\title{
D/HD transition in Photon Dominated Regions (PDR)
}

\author{
F. Le Petit, E. Roueff, and J. Le Bourlot
}

\author{
LUTH and FRE2462 du CNRS, Observatoire de Paris, Place J. Janssen, 92195 Meudon Cedex, France \\ e-mail: Franck.Lepetit@obspm.fr, Jacques.1ebourlot@obspm.fr
}

Received 10 July 2001 / Accepted 15 May 2002

\begin{abstract}
We present the basic features of a steady state chemical model of Photon Dominated Regions (PDR), where the deuterium chemistry is explicitly introduced. The model is an extension of a previous PDR model (Abgrall et al. 1992; Le Bourlot et al. 1993; Le Bourlot 2000) in which the microscopic processes relative to HD have been incorporated. The $J$-dependent photodissociation probabilities have been calculated and included in the statistical equilibrium of the rotational levels of HD where the latest collision molecular data are also introduced. The thermal balance is calculated from the equilibrium between the different heating and cooling processes. We introduce a standard model of density $n_{\mathrm{H}}=500 \mathrm{~cm}^{-3}$ embedded in the Interstellar Standard Radiation Field (ISRF) from which we derive the main properties of HD in PDR. The D/HD transition does not depend only on the density, radiation field but also on the chemical processes and especially on the dust formation efficiency. In standard radiation field conditions, the $\mathrm{D} / \mathrm{HD}$ transition occurs in a narrow range of visual extinctions as long as density is less than $1000 \mathrm{~cm}^{-3}$ and HD is formed through the $\mathrm{D}^{+}+\mathrm{H}_{2}$ reaction. At higher densities a logarithmic dependence of the location of the transition is derived. The model is applied both to ultraviolet absorption observations from the ground rotational state of HD performed in diffuse and translucent clouds and infrared emission detectable at high densities and for high ultraviolet radiation fields coming from the bright surrounding stars.
\end{abstract}

Key words. astrochemistry - molecular processes - ISM: molecules

\section{Introduction}

Since deuterium has been formed only at the early beginning of the universe, the elemental deuterium to hydrogen ratio is one pivotal parameter to understand the evolution of astrophysical media. In the interstellar medium (ISM), deuterium may be present in atomic but also in molecular form and over 20 single D-bearing molecules and two doubly deuterated ones have been found in cold molecular clouds and star forming regions (Roueff et al. 2000; Loinard et al. 2000). Last but not least, the triply deuterated ammonia has been detected towards two dense cold clouds (Lis et al. 2002; van der Tak et al. 2002). However, chemical fractionation processes and mantle desorption from grains take place in these molecular clouds and it is not straightforward to derive the elemental deuterium abundance from such observations (Roberts \& Millar 2000a,b).

Atomic deuterium has first been observed with the Copernicus satellite in the local interstellar medium and in diffuse clouds in absorption towards bright stars where HD has also been detected (see Lemoine et al. 1999 for a review). The successful launch of the FUSE (Far Ultraviolet Spectroscopy Explorer) mission allows to search for molecular HD towards fainter sources, in translucent clouds which are intermediate between diffuse and molecular clouds. FUSE detections

Send offprint requests to: E. Roueff, e-mail: Evelyne.Roueff@obspm.fr of interstellar HD have been reported in a variety of galactic lines of sight (Ferlet et al. 2000; Rachford et al. 2001; Lacour et al. 2002; Boissé et al. 2002) and in the LMC (Bluhm \& De Boer 2002). Moreover, the short wavelength spectrograph (SWS) of the ISO (Infrared Space Observatory) satellite has allowed the observations of the pure rotational emission transitions of HD at $112 \mu \mathrm{m}(J=1-0)$ (Wright et al. 1999) in the Orion Bar and at $19.43 \mu \mathrm{m}(J=6-5)$ (Bertoldi et al. 1999) in Orion KL. Finally, the $2.64 \mu \mathrm{m},(1-0) \mathrm{R}(5)$ line of HD has also been detected in Orion Peak 1 by Ramsay Howat et al. (2002) at UKIRT. These two last detections have been performed in shocked regions where the present paper does not apply.

From the modelling point of view, Black \& Dalgarno (1973) have first considered the atomic to molecular transition of deuterium in diffuse clouds to interpret Copernicus observations. Viala et al. (1988a) have developed a more elaborate model of diffuse clouds in the same context where the photochemical equilibrium is solved at a fixed temperature. Meanwhile, the rotational and rovibrational collisional excitation of $\mathrm{HD}$ by $\mathrm{H}$, He and $\mathrm{H}_{2}$ has been studied with quantal close coupling techniques and new molecular potential surfaces (Roueff \& Zeippen 1999, 2000; Flower \& Roueff 1999). Transition and photodissociation probabilities of $\mathrm{H}_{2}$ and HD have been improved (Abgrall et al. 2000; Abgrall \& Roueff 2002) taking into account the rotational coupling and new transition moments calculations. 
We present in this paper an updated PDR model where the chemistry as well as the microscopic processes of HD have been introduced. The chemical and statistical equilibrium are solved together with the thermal balance and the most recent molecular data have been included. The basic features of the model are discussed in Sect. 2. In Sect. 3, a standard model is presented and the influence of the various physical parameters (density, intensity of the incident radiation field, rate of formation of HD on dust) is considered. Comparison with observations are reviewed in Sect. 4.

\section{PDR model}

\subsection{General features}

The present study is a straightforward extension of the steady state PDR model of Abgrall et al. (1992), Le Bourlot et al. (1993) and Le Bourlot (2000). We consider a steady state model of an interstellar cloud as an infinite slab of gas and dust irradiated by an ultraviolet radiation field impinging on one side of the cloud. The incoming ultraviolet radiation field is expressed in units of the ISRF model of Draine (1978). The radiative transfer is solved in decoupling the continuum extinction due to dust and gas and the absorption in lines of $\mathrm{H}_{2}$, $\mathrm{HD}$ and $\mathrm{CO}$. In addition, we use the approximation introduced by Federman et al. (1979) to estimate the self-absorption in the dissociating lines of $\mathrm{H}_{2}$, $\mathrm{HD}$ and $\mathrm{CO}$, and we neglect the overlap between the lines of $\mathrm{H}_{2}$ and other molecules. This approximation reproduces the main physical properties as shown by Abgrall et al. (1992) and allows rapid computations.

The abundances of 139 chemical species linked by a network of 1416 chemical reactions are computed as a function of the visual extinction. These species consist of $\mathrm{H}, \mathrm{D}, \mathrm{He}, \mathrm{O}, \mathrm{C}$, $\mathrm{N}, \mathrm{S}$ and a representative metal $\mathrm{M}$. The elemental depletions are taken from the recent HST observations and the gas phase abundances are displayed in Table 1. Since the elemental abundance of deuterium is not well known, it can be considered as a free parameter with a value close to $1.6 \times 10^{-5}$ (Linsky et al. 1995), the mean value in the local ISM. In the present model, we have arbitrarily fixed it at $2 \times 10^{-5}$.

The chemical equations are solved in parallel to the excitation processes (see next section) and include $J$-dependent photodissociation rates of $\mathrm{H}_{2}, \mathrm{HD}$ and $\mathrm{CO}$.

Thermal balance is solved as discussed in Le Bourlot et al. (1993). The heating due to photoelectric effect on grains has been updated following Le Bourlot (2000) where the actual magnitude of the ISRF is calculated at the different visual extinctions. The charge of the grains and the resulting photoelectric heating are functions of the grain size, following the derivation of Bakes \& Tielens (1994). Cooling processes are principally due to radiative emission following excitation of abundant atoms and molecules. We derive the level populations of the various coolants $\left(\mathrm{C}, \mathrm{C}^{+}, \mathrm{O}, \mathrm{H}_{2}, \mathrm{HD}, \mathrm{CO}, \mathrm{CS}\right.$, $\mathrm{HCO}^{+}$) by solving the corresponding statistical equilibrium. Then we compute the local emissivities corresponding to the cooling emission, taking into account possible optical depths effects.
Table 1. Elemental gas phase abundances.

\begin{tabular}{lr}
\hline \hline $\mathrm{D} / \mathrm{H}^{(1)}$ & $2 \times 10^{-5}$ \\
$\mathrm{O} / \mathrm{H}^{(2)}$ & $3.19 \times 10^{-4}$ \\
$\mathrm{He} / \mathrm{H}$ & $1 \times 10^{-1}$ \\
$\mathrm{~N} / \mathrm{H}^{(3)}$ & $7.5 \times 10^{-5}$ \\
$\mathrm{C} / \mathrm{H}^{(4)}$ & $1.32 \times 10^{-4}$ \\
$\mathrm{~S} / \mathrm{H}^{(4)}$ & $1.85 \times 10^{-6}$ \\
$\mathrm{M} / \mathrm{H}$ & $1.5 \times 10^{-8}$ \\
\hline
\end{tabular}

${ }^{1}$ free parameter in our model.

${ }^{2}$ Meyer et al. (1998).

${ }^{3}$ Meyer et al. (1997).

${ }^{4}$ Savage et Sembach (1996).

At the edge of the cloud, all atoms with ionization energies smaller than $13.6 \mathrm{eV}$, the ionization threshold of atomic hydrogen, are mainly ionic and the others are predominantly neutral. Formation of $\mathrm{H}_{2}$ takes place on grains in competition with destruction by photodissociation. The medium becomes progressively molecular as the visual extinction increases and photodissociation probability decreases, (van Dishoeck \& Black 1986; Abgrall et al. 1992). The $\mathrm{H} / \mathrm{H}_{2}$ and the $\mathrm{C}^{+} / \mathrm{C} / \mathrm{CO}$ transitions in the envelopes of interstellar clouds have been the subject of many modelling studies (van Dishoeck \& Black 1988; Viala et al. 1988b; Hollenbach \& Tielens 1999), including those undertaken in our own group (Le Bourlot et al. 1993). There have been much less studies on the D/HD transition (Black \& Dalgarno 1973; Viala et al. 1988b), regarding to the very few observational data available up to now.

The $\mathrm{H}_{2}$ molecule and its deuterated substitute, $\mathrm{HD}$, present very similar properties. The photodissociation mechanisms resulting from fluorescence towards the continuum of the ground electronic state following discrete absorption transitions in the Lyman and Werner band systems are identical. However, the substitution of a proton by a deuteron, leads to some significant differences with $\mathrm{H}_{2}$. First, HD possesses a small permanent dipole moment of $8.3 \times 10^{-4}$ Debye due to the shift between the center of mass and the center of charge. So electric dipole transitions, whose probabilities have been calculated by Abgrall et al. (1982), occur between rovibrational levels within the ground state, whereas only electric quadrupole transitions are possible for $\mathrm{H}_{2}$. Secondly, the chemical formation processes of $\mathrm{HD}$ and $\mathrm{H}_{2}$ are different as discussed in Sect. 2.3.

The parameters of the models are the proton density $n_{\mathrm{H}}$ (in $\mathrm{cm}^{-3}$ ), the cosmic ray ionization rate of $\mathrm{H}_{2}, \zeta$ (in s${ }^{-1}$ ), the Doppler turbulent parameter $b$ (in $\mathrm{km} \mathrm{s}^{-1}$ ) and the scaling factor $\chi$ of the ISRF in Draine's unit. The grain properties play a considerable role, both for the chemistry (formation of $\mathrm{H}_{2}$ and HD) and for the thermal balance. In the actual model, we take the standard galactic values of the mass per unit volume, the albedo and asymmetry factor of the grains $(g)$ as well as the dust to gas mass ratio $(G)$. We introduce the size distribution of the grains as a power law with exponent 3.5 following Mathis et al. (1977) and an analytic dependence of the extinction curve from the UV to the visible using the Fitzpatrick \& Massa (1990) expansion coefficients. The corresponding values 
Table 2. Standard values of the grain parameters.

\begin{tabular}{cc}
\hline \hline albedo & 0.42 \\
$g$ & 0.6 \\
$\rho\left(\mathrm{g} / \mathrm{cm}^{-3}\right)$ & 3 \\
$G$ & 0.01 \\
$r_{\min }(\mu \mathrm{m})$ & 0.003 \\
$r_{\max }(\mu \mathrm{m})$ & 0.3 \\
$N_{H} / A_{V}(1)$ & $1.87 \times 10^{21}$ \\
\hline$c 1$ & -0.38 \\
$c 2$ & 0.74 \\
$c 3$ & 3.96 \\
$c 4$ & 0.26 \\
$\gamma$ & 1.05 \\
$\lambda_{0}^{-1}$ & 4.59 \\
\hline
\end{tabular}

(1) from Bohlin et al. (1978) expressed in $\mathrm{cm}^{-2} \mathrm{mag}^{-1}$. Values in the second part of the chart are the coefficients of the galactic extinction curve parameterized by Fitzpatrick \& Massa (1990).

as well as the relation between the total proton column density and the visual extinction are given in Table 2.

\subsection{Excitation of $H D$}

We solve the statistical equilibrium of the nine first rotational levels of HD, with energy terms below the first excited vibrational level. The corresponding energies are given in Table 3.

We consider three possible contributions to the excitation: radiative pumping, collisions and chemical formation. Radiative pumping involves absorption by the electronic Lyman and Werner transitions. The excited B and C electronic states decay towards the discrete rovibrational levels of the ground state or towards the continuum, which leads to dissociation of the HD molecule (occurs in approximately $15 \%$ of cases). The excited rovibrational levels of the ground electronic state cascade towards the rotational ground state via faint electric dipole transitions with $|\Delta J|=1$ selection rule as calculated by Abgrall et al. (1982). Collisional excitations occur with the most abundant species in the cloud: $\mathrm{H}, \mathrm{He}, \mathrm{H}_{2}$ and electrons. Finally, the chemical formation processes of HD may also contribute to its excitation. We apply the cascade formalism introduced first by Black \& Dalgarno (1976) for molecular hydrogen and extended to $\mathrm{HD}$ and $\mathrm{C}_{2}$ respectively by Viala et al. (1988b) and Le Bourlot et al. (1987). We assume that the rovibrational levels of the ground electronic state with energies higher than $4445 \mathrm{~K}$ (corresponding to the level $v=0, J=8$ ) are only decaying via spontaneous radiative transitions. Then, the equations governing the time evolution of the abundances of the nine first rotational levels of $\mathrm{HD}, n_{0 J}$, can be written:

$$
\frac{\mathrm{d} n_{0 J}}{\mathrm{~d} t}=\left(\frac{\mathrm{d} n_{0 J}}{\mathrm{~d} t}\right)_{\text {rad. }}+\left(\frac{\mathrm{d} n_{0 J}}{\mathrm{~d} t}\right)_{\text {coll. }}+\left(\frac{\mathrm{d} n_{0 J}}{\mathrm{~d} t}\right)_{\text {chemistry }} .
$$$$
\text { (1) }\left(\frac{\mathrm{d} n_{0 J}}{\mathrm{~d} t}\right)_{\text {chemistry }}=\sum_{m} k_{m} n_{X} n_{Y} p_{0 j}^{m}-\sum_{l} k_{l} n_{Z} n_{0 J}
$$

Table 3. Energies of the first levels of HD.

\begin{tabular}{clc}
\hline \hline \multicolumn{2}{c}{ Level } & Energy in Kelvin \\
\hline$v=0$ & $J=0$ & 0. \\
$v=0$ & $J=1$ & 128.38 \\
$v=0$ & $J=2$ & 384.26 \\
$v=0$ & $J=3$ & 765.89 \\
$v=0$ & $J=4$ & 1270.7 \\
$v=0$ & $J=5$ & 1895.4 \\
$v=0$ & $J=6$ & 2635.9 \\
$v=0$ & $J=7$ & 3487.5 \\
$v=0$ & $J=8$ & 4445.3 \\
$v=1$ & $J=0$ & 5226.7 \\
\hline
\end{tabular}

The equation describing the excitation by radiative processes can be written as:

$$
\begin{aligned}
\left(\frac{\mathrm{d} n_{0 J}}{\mathrm{~d} t}\right)_{\mathrm{rad} .}= & d_{0 J}+D_{0 J}-n_{0 J} \sum_{J^{\prime}<J} A_{0 J, 0 J^{\prime}} \\
& -n_{0 J} \sum_{B, C} \sum_{v^{\prime} J^{\prime}} B_{0 J, v^{\prime} J^{\prime}} \int_{912}^{\infty} u(\lambda) \cdot \phi_{0 J, v^{\prime} J^{\prime}}(\lambda) \mathrm{d} \lambda
\end{aligned}
$$

where $u(\lambda)$ is the density of the energy of the interstellar ultraviolet radiation field and $\phi_{0 J, v^{\prime} J^{\prime}}$ is the profile of the line for the considered transition. $B$ and $C$ refer to the excited electronic states, and $v^{\prime} J^{\prime}$ labels a rovibrational level belonging to one of these excited electronic states. $d_{0 J}$ is the summation of the direct radiative de-excitation terms from the electronic excited levels $v^{\prime} J^{\prime}$ toward level $0 J$ of the ground electronic state and $D_{0 J}$ is the total de-excitation term due to the radiative cascades inside the ground electronic state:

$d_{0 J}=\sum_{B, C} \sum_{v^{\prime} j^{\prime}} A_{v^{\prime} J^{\prime}, 0 J} n_{v^{\prime} J^{\prime}}$
$D_{0 J}=\sum_{v^{\prime \prime} J^{\prime \prime}} A_{v^{\prime \prime} J^{\prime \prime}, 0 J} n_{v^{\prime \prime} J^{\prime \prime}}$

Levels $v^{\prime \prime} J^{\prime \prime}$ and $0 J$ belong to the ground electronic state with $E\left(v^{\prime \prime} J^{\prime \prime}\right)>E(0 J)$.

The contribution of collisions to the rotational populations of HD is:

$$
\begin{aligned}
\left(\frac{\mathrm{d} n_{0 J}}{\mathrm{~d} t}\right)_{\text {coll. }}= & \sum_{q} \sum_{J^{\prime \prime}=0}^{J=8} K_{0 J^{\prime \prime}, 0 J^{\prime \prime}}^{q} n_{J^{\prime \prime}} n_{q} \\
& -\sum_{q} \sum_{J^{\prime \prime}=0}^{J=8} K_{0 J, 0 J^{\prime \prime}}^{q} n_{0 J} n_{q} .
\end{aligned}
$$

In this equation, $K_{0 J^{\prime \prime}, 0 J}^{q}$ is the collision rate of HD with a perturber $q$ for the transition from the level $0 J^{\prime \prime}$ toward the level $0 J$. In the present case, we consider as possible perturbers, electrons, $\mathrm{H}, \mathrm{He}$ and $\mathrm{H}_{2}$.

Finally, the contribution of chemistry to the excitation of HD gives:

$\left(\frac{\mathrm{d} n_{0 J}}{\mathrm{~d} t}\right)_{\text {chemistry }}=\sum_{m} k_{m} n_{X} n_{Y} p_{0 j}^{m}-\sum_{l} k_{l} n_{Z} n_{0 J}$ 
where $n_{X}, n_{Y}$ and $n_{Z}$ are the densities of the reagents, $k_{m}$ is the rate coefficient of the $m$ chemical reaction and $p_{0 J}^{m}$ is the probability to produce HD in the level $0 J$ through this reaction.

The various contributions are calculated with the most recent molecular data. Previous transition probabilities in the Lyman and Werner band systems have been calculated by Allison \& Dalgarno (1970) in a single state approximation and assuming that $J=0$. We introduce the values calculated by Abgrall \& Roueff (2002) where the rotational coupling and centrifugal barrier potential are taken into account in the resolution of the Schrödinger equation. The rovibrational collisional excitation and de-excitation rate coefficients of $\mathrm{H}_{2}$ and $\mathrm{HD}$ by $\mathrm{H}, \mathrm{He}$ and $\mathrm{H}_{2}$, are those reported in Flower et al. (2000). They are expressed as analytical functions of the temperature and can be found at url: http: //ccp7.dur.ac.uk.

The critical density (the minimum density required to reach thermodynamical equilibrium in a two-level approximation) is the ratio between the Einstein spontaneous de-excitation rate and the collisional de-excitation rate coefficient:

$n_{\text {crit }}(J)=\frac{A_{J, J-1}}{K_{J, J-1}}$.

In this equation, $K_{J, J-1}$, is the weighted sum of the collisional de-excitation rate coefficients of all the perturbers:

$K_{J, J-1}=\sum_{q} \frac{n(q)}{n} K_{J, J-1}^{q}$

where $n$ is the value of the actual density.

We have computed the critical densities of levels $J=1$ to $J=8$ of HD in function of the temperature, both for an atomic gas and a molecular gas. Results are presented in Tables 4 and 5 . We note that the values are typically larger than $1000 \mathrm{~cm}^{-3}$ for $J=1$ and that they decrease with temperature, reflecting the temperature dependence of the collisional rate coefficients. Critical density values increase significantly with $J$. Chemical excitation seems unlikely to compete with collisional or radiative processes.

\subsection{Chemistry of $H D$}

The gas phase chemistry of HD has been discussed in the context of primordial clouds by Galli \& Palla (1998) and Stancil et al. (1998). We have introduced the corresponding reactions in the present model, in addition to the previous updated network that has been used in the context of shock chemistry (Pineau des Forêts et al. 1989).

Whereas $\mathrm{H}_{2}$ formation occurs only on dust, the formation of HD may involve surface as well as gas phase processes. Formation on dust is assumed to occur similarly to $\mathrm{H}_{2}$. A first step involves the adsorption of $\mathrm{D}$ and $\mathrm{H}$ at the surface of the grains. The two adsorbed atoms meet then on this surface and gaseous $\mathrm{HD}$ is desorbed. We note $\mathrm{D}$ : and $\mathrm{H}$ : respectively the adsorbed deuterium and adsorbed hydrogen.

$\begin{cases}\mathrm{D}+\text { dust } \underset{\mathrm{ads}}{\longrightarrow} \mathrm{D}: & k_{\mathrm{ads}}=s<n_{\mathrm{gr}} \sigma>v \quad\left(\mathrm{~s}^{-1}\right) \\ \mathrm{H}+\text { dust } \stackrel{k_{\mathrm{ads}}^{\prime}}{\longrightarrow} \mathrm{H}: & k_{\mathrm{ads}}^{\prime}=s^{\prime}<n_{\mathrm{gr}} \sigma>v^{\prime} \quad\left(\mathrm{s}^{-1}\right) \\ \mathrm{D}:+\mathrm{H}: \stackrel{k_{\mathrm{dust}}}{\longrightarrow} \mathrm{HD} & \end{cases}$
Table 4. Critical densities $n_{\text {crit }}$ in $\mathrm{cm}^{-3}$ of HD levels in a neutral gas mixture of $\mathrm{H}$ and $\mathrm{He}$ with a fractional abundance of He equals to 0.1 .

\begin{tabular}{c|ccccc}
\hline \hline Level & $T=10 \mathrm{~K}$ & $50 \mathrm{~K}$ & $100 \mathrm{~K}$ & $200 \mathrm{~K}$ & $500 \mathrm{~K}$ \\
\hline$J=1$ & $6.1 \times 10^{3}$ & $4.5 \times 10^{3}$ & $3.3 \times 10^{3}$ & $2.0 \times 10^{3}$ & $9.4 \times 10^{2}$ \\
$J=2$ & $5.8 \times 10^{4}$ & $4.1 \times 10^{4}$ & $2.9 \times 10^{4}$ & $1.7 \times 10^{4}$ & $7.1 \times 10^{3}$ \\
$J=3$ & $2.3 \times 10^{5}$ & $1.6 \times 10^{5}$ & $1.1 \times 10^{5}$ & $6.3 \times 10^{4}$ & $2.5 \times 10^{4}$ \\
$J=4$ & $7.4 \times 10^{5}$ & $5.0 \times 10^{5}$ & $3.3 \times 10^{5}$ & $1.8 \times 10^{5}$ & $6.8 \times 10^{4}$ \\
$J=5$ & $3.7 \times 10^{6}$ & $2.1 \times 10^{6}$ & $1.2 \times 10^{6}$ & $5.2 \times 10^{5}$ & $1.5 \times 10^{5}$ \\
$J=6$ & $7.9 \times 10^{6}$ & $4.5 \times 10^{6}$ & $2.6 \times 10^{6}$ & $1.1 \times 10^{6}$ & $3.0 \times 10^{5}$ \\
$J=7$ & $1.5 \times 10^{7}$ & $8.8 \times 10^{6}$ & $5.0 \times 10^{6}$ & $2.2 \times 10^{6}$ & $5.7 \times 10^{5}$ \\
$J=8$ & $2.1 \times 10^{7}$ & $1.3 \times 10^{7}$ & $8.2 \times 10^{6}$ & $3.8 \times 10^{6}$ & $1.0 \times 10^{6}$ \\
\hline
\end{tabular}

Table 5. Critical densities $n_{\text {crit }}$ in $\mathrm{cm}^{-3}$ of HD levels in a neutral gas mixture of $\mathrm{H}_{2}$ and $\mathrm{He}$ with the number density ratio of $\mathrm{He}$ to $\mathrm{H}_{2}$ of 0.2 . Ortho and para populations of $\mathrm{H}_{2}$ have been taken at the thermal equilibrium.

\begin{tabular}{c|ccccc}
\hline \hline Level & $T=10 \mathrm{~K}$ & $50 \mathrm{~K}$ & $100 \mathrm{~K}$ & $200 \mathrm{~K}$ & $500 \mathrm{~K}$ \\
\hline$J=1$ & $3.3 \times 10^{3}$ & $2.6 \times 10^{3}$ & $2.0 \times 10^{3}$ & $1.4 \times 10^{3}$ & $8.0 \times 10^{2}$ \\
$J=2$ & $2.0 \times 10^{4}$ & $1.7 \times 10^{4}$ & $1.4 \times 10^{4}$ & $1.0 \times 10^{4}$ & $5.7 \times 10^{3}$ \\
$J=3$ & $9.1 \times 10^{4}$ & $7.2 \times 10^{4}$ & $5.6 \times 10^{4}$ & $3.9 \times 10^{4}$ & $2.0 \times 10^{4}$ \\
$J=4$ & $3.6 \times 10^{5}$ & $2.8 \times 10^{5}$ & $2.0 \times 10^{5}$ & $1.2 \times 10^{5}$ & $5.5 \times 10^{4}$ \\
$J=5$ & $8.1 \times 10^{5}$ & $6.2 \times 10^{5}$ & $5.1 \times 10^{5}$ & $3.4 \times 10^{5}$ & $1.4 \times 10^{5}$ \\
$J=6$ & $5.9 \times 10^{6}$ & $3.6 \times 10^{6}$ & $2.1 \times 10^{6}$ & $9.4 \times 10^{5}$ & $2.8 \times 10^{5}$ \\
$J=7$ & $1.3 \times 10^{7}$ & $8.7 \times 10^{6}$ & $5.1 \times 10^{6}$ & $2.3 \times 10^{6}$ & $6.1 \times 10^{5}$ \\
$J=8$ & $3.6 \times 10^{7}$ & $2.2 \times 10^{7}$ & $1.3 \times 10^{7}$ & $5.3 \times 10^{6}$ & $1.2 \times 10^{7}$ \\
\hline
\end{tabular}

In these equations, the adsorption rate coefficient $k_{\text {ads }}$ can be expressed as the product of the sticking coefficient, $s$, the relative velocity of the atom to the grain, $v$, and the average over the size distribution of the grains of the product of the geometric cross section of the grains, $\sigma$, by the density of grains $n_{\mathrm{gr}}$. The sticking coefficient $s$ is a decreasing function of temperature (Hollenbach \& Mc Kee 1979). We assume here that the sticking coefficient equals 1 when $T$ is smaller than $10 \mathrm{~K}$ and varies as $T^{-1 / 2}$ at larger temperatures. The adsorption rate coefficient becomes then independent on the temperature for values larger than $10 \mathrm{~K}$ since the atomic velocity is proportional to $\sqrt{T}$. The formation rate of $\mathrm{H}_{2}$ and $\mathrm{HD}$ on grains (respectively $\mathcal{F} \mathcal{R}\left(\mathrm{H}_{2}\right)$ and $\mathcal{F} \mathcal{R}(\mathrm{HD})$ ) can be expressed respectively as:

$$
\begin{aligned}
& \left.\frac{\mathrm{d} n\left(\mathrm{H}_{2}\right)}{\mathrm{d} t}\right|_{\text {dust form. }}=\frac{1}{2} k_{\mathrm{ads}}^{\prime} n(\mathrm{H})=\mathcal{F} \mathcal{R}\left(\mathrm{H}_{2}\right) n(\mathrm{H}) \\
& \left.\frac{\mathrm{d} n(\mathrm{HD})}{\mathrm{d} t}\right|_{\text {dust form. }}=k_{\mathrm{ads}} n(\mathrm{D})=\mathcal{F} \mathcal{R}(\mathrm{HD}) n(\mathrm{D}) .
\end{aligned}
$$

Following Le Bourlot et al. (1995) and Roueff (2002), $k_{\text {ads }}$ can be expressed by:

$k_{\mathrm{ads}}=s \cdot \frac{3}{4} \cdot 1.4 \cdot m_{\mathrm{H}} \frac{G}{\rho} \frac{1}{\sqrt{r_{\max } \cdot r_{\min }}} v n_{\mathrm{H}}$

with $m_{\mathrm{H}}$ the mass of the atom of hydrogen and $n_{\mathrm{H}}$ the density of protons. Using the parameters in Table 2, we obtain:

$\begin{array}{ll}\mathcal{F} \mathcal{R}\left(H_{2}\right)=4.4 \times 10^{-17} \times n_{\mathrm{H}} & \left(\mathrm{s}^{-1}\right) \\ \mathcal{F} \mathcal{R}(H D) & =6.3 \times 10^{-17} \times n_{\mathrm{H}} \quad\left(\mathrm{s}^{-1}\right) .\end{array}$ 
These values are very close to the first derivation of the formation rate of $\mathrm{H}_{2}$ by Jura (1975) from Copernicus observations in diffuse clouds and to the one deduced by Gry et al. (2002) from FUSE observations.

As soon as $\mathrm{H}_{2}$ is present, gas phase chemical processes may contribute efficiently to the formation of HD. In diffuse clouds, the relevant reaction is:

$\mathrm{D}^{+}+\mathrm{H}_{2} \stackrel{k_{1}}{\rightarrow} \mathrm{H}^{+}+\mathrm{HD} \quad k_{1}=2 \times 10^{-9}\left(\mathrm{~cm}^{3} \mathrm{~s}^{-1}\right)$.

The reverse reaction involving an endothermicity of about 464 Kelvin is not efficient in cold regions.

On the other hand, in dense PDRs illuminated by a strong radiation field, the temperature may reach several hundred Kelvin. Then the neutral neutral reaction between $\mathrm{D}$ and $\mathrm{H}_{2}$, may become the most efficient process of formation of HD:

$\mathrm{D}+\mathrm{H}_{2} \stackrel{k_{2}}{\rightarrow} \mathrm{H}+\mathrm{HD} \quad k_{2}=7.5 \times 10^{-11} \mathrm{e}^{-3820 / T}\left(\mathrm{~cm}^{3} \mathrm{~s}^{-1}\right)$.

The corresponding chemical formation rates are respectively:

$$
\begin{aligned}
\left.\frac{\mathrm{d} n(\mathrm{HD})}{\mathrm{d} t}\right|_{D^{+}+H_{2}} & =k_{1} n\left(\mathrm{D}^{+}\right) n\left(\mathrm{H}_{2}\right) \\
& =2.1 \times 10^{-9} n\left(\mathrm{D}^{+}\right) n\left(\mathrm{H}_{2}\right) \\
\left.\frac{\mathrm{d} n(\mathrm{HD})}{\mathrm{d} t}\right|_{D+H_{2}} & =k_{2} n(\mathrm{D}) n\left(\mathrm{H}_{2}\right) \\
= & 7.5 \times 10^{-11} \mathrm{e}^{-3820 / T} n(\mathrm{D}) n\left(\mathrm{H}_{2}\right) .
\end{aligned}
$$

It can be shown that the abundance of $\mathrm{D}^{+}$is almost independent on the density. Then, the rate of formation of HD through the reaction between $\mathrm{D}^{+}$and $\mathrm{H}_{2}$ is proportional to the density (Eq. (11)). This is no more the case when formation of HD on dust or when the reaction between $\mathrm{D}$ and $\mathrm{H}_{2}$ takes place. The rate of formation of $\mathrm{HD}$ is then proportional to the square of the density (Eqs. $(7,12))$. We infer that in diffuse clouds, the main route of formation of HD is the reaction in gas phase between $\mathrm{D}^{+}$and $\mathrm{H}_{2}$ as noted previously by Black \& Dalgarno (1973). However, formation on dust or formation by the reaction between neutral atomic deuterium and molecular hydrogen becomes competitive and even dominant when the density is larger than about $5 \times 10^{3} \mathrm{~cm}^{-3}$. In such clouds, formation of HD occurs on dust at low temperatures and by the gas phase reaction between $\mathrm{D}$ and $\mathrm{H}_{2}$ for high temperatures. Nevertheless, we should keep in mind that the rate of formation on dust is not well known whereas a relative good agreement between different studies is achieved for the reaction rate coefficient involving $D$ and $\mathrm{H}_{2}$.

At the edge of the cloud and up to regions where atomic deuterium becomes molecular, destruction of HD occurs mainly by photodissociation. Nevertheless, in the case of a dense and warm molecular cloud, the reaction between HD and $\mathrm{H}$, which is the reverse reaction of Eq. (10), is not negligible:

$\mathrm{HD}+\mathrm{H} \stackrel{k_{2}^{\prime}}{\longrightarrow} \mathrm{H}_{2}+\mathrm{D} \quad k_{2}^{\prime}=7.5 \times 10^{-11} \mathrm{e}^{-4240 / T}\left(\mathrm{~cm}^{3} \mathrm{~s}^{-1}\right)$.

Once photo-processes are negligible, destruction by chemical processes becomes dominant. Chemical fractionation of deuterated molecules occurs as reviewed recently by Roberts \& Millar (2000a,b) and Le Petit \& Roueff (2002). The important step towards other deuterated molecules is the proton transfer reaction of $\mathrm{H}_{3}^{+}$in the reaction with $\mathrm{HD}$ forming $\mathrm{H}_{2} \mathrm{D}^{+}$:

$\mathrm{H}_{3}^{+}+\mathrm{HD} \stackrel{k}{\rightarrow} \mathrm{H}_{2} \mathrm{D}^{+}+\mathrm{H}_{2} \quad k=3.5 \times 10^{-10}\left(\mathrm{~cm}^{3} \mathrm{~s}^{-1}\right)$

The reverse reaction is endothermic when $\mathrm{H}_{2}$ is in its ground rotational state $J=0$ and its efficiency depends on the amount of ortho $\mathrm{H}_{2}$ present in the environment (Gerlich et al. 2002).

\section{Results}

\subsection{Standard model}

We consider a standard chemical model of translucent cloud where the elemental gas phase abundances and dust properties are given in Tables 1 and 2. In addition, we take the following physical parameters: density $n_{H}=500 \mathrm{~cm}^{-3}$, cosmic ray ionization rate $\zeta=5 \times 10^{-17} \mathrm{~s}^{-1}$, turbulent velocity $b=2 \mathrm{~km} \mathrm{~s}^{-1}$ and an incident radiation field corresponding to the $\operatorname{ISRF}(\chi=1)$.

Figure 1 displays on its upper panel, the photodissociation rates of $\mathrm{H}_{2}$ and $\mathrm{HD}$ as a function of $A_{V}$ and on its lower panel, the normalized abundances, on one hand, of $\mathrm{H}$ and $\mathrm{H}_{2}$, and on the other hand, of $\mathrm{D}$ and $\mathrm{HD}$ as a function of $A_{V}$. The photodissociation rates (in s ${ }^{-1}$ ) of $\mathrm{H}_{2}$ and $\mathrm{HD}$ are the same when $A_{V}=0$. This reflects the identity of the microscopic processes. The respective decrease of the photodissociation rates of $\mathrm{H}_{2}$ and $\mathrm{HD}$ is due to the absorption of the radiation field by grains and to the self-shielding. Self-shielding becomes efficient as soon as the optical depths of the discrete absorbing transitions is of the order of 1. The optical depth in the center of the lines of $\mathrm{H}_{2}$ or $\mathrm{HD}, \tau_{\mathrm{H}_{2} / \mathrm{HD}}$, is given by:

$$
\begin{aligned}
\tau_{\mathrm{H}_{2} / \mathrm{HD}}= & \frac{\pi e^{2}}{m_{\mathrm{e}} c} f_{\mathrm{lu}} \frac{\lambda_{0}}{\sqrt{\pi} \cdot \sqrt{v_{\text {turb }}^{2}+2 k T / m_{\mathrm{H}_{2} / \mathrm{HD}}}} \\
& \times N_{\mathrm{l}\left(\mathrm{H}_{2} / \mathrm{HD}\right)} \\
= & \frac{1.497 \times 10^{-2} \lambda_{0} f_{\mathrm{lu}}}{\sqrt{v_{\text {turb }}^{2}+2 k T / m_{\mathrm{H}_{2} / \mathrm{HD}}}} N_{1}\left(\mathrm{H}_{2} / \mathrm{HD}\right) \quad(C G S)
\end{aligned}
$$

with $f_{\mathrm{lu}}$ the oscillator strength of the Lyman and Werner band systems, $\lambda_{0}$ the wavelength in the center of the line, $\mathrm{N}_{l}\left(\mathrm{H}_{2} / \mathrm{HD}\right)$, the column density of $\mathrm{H}_{2}$ or $\mathrm{HD}$ in the lower level, $m_{\mathrm{e}}$ the mass of the electron and $\mathrm{m}_{\mathrm{H}_{2} / \mathrm{HD}}$ the mass of $\mathrm{H}_{2}$ or $\mathrm{HD}$. As oscillator strengths of the Lyman and Werner band systems are typically of the order of $10^{-3}$ and the wavelength of the order of $100 \mathrm{~nm}$, the optical depth reaches a value of 1 when the column density of $\mathrm{H}_{2}$ or HD is about $10^{15} \mathrm{~cm}^{-2}$. As deuterium is typically $10^{-5}$ times less abundant than hydrogen, self-shielding effects take place at larger visual extinctions; the formation of HD occurs deeper in the cloud than $\mathrm{H}_{2}$. The comparison of both panels of Fig. 1 shows that the $\mathrm{H} / \mathrm{H}_{2}$ transition and the $\mathrm{D} / \mathrm{HD}$ one are strongly linked to the photodissociation rates.

Temperature profile, obtained as a result of the thermal equilibrium in the gas phase, is displayed in function of $A_{V}$ in Fig. 2. Temperature ranges from 70 Kelvin at the edge of the cloud to 10 Kelvin at visual extinctions larger than 2 . The $\mathrm{H} / \mathrm{H}_{2}$ transition takes place for $A_{V} \simeq 3 \times 10^{-3}$ at about 70 Kelvin whereas the $\mathrm{D} / \mathrm{HD}$ transition occurs at $A_{V} \simeq 0.5$ where the temperature is close to $50 \mathrm{~K}$. 

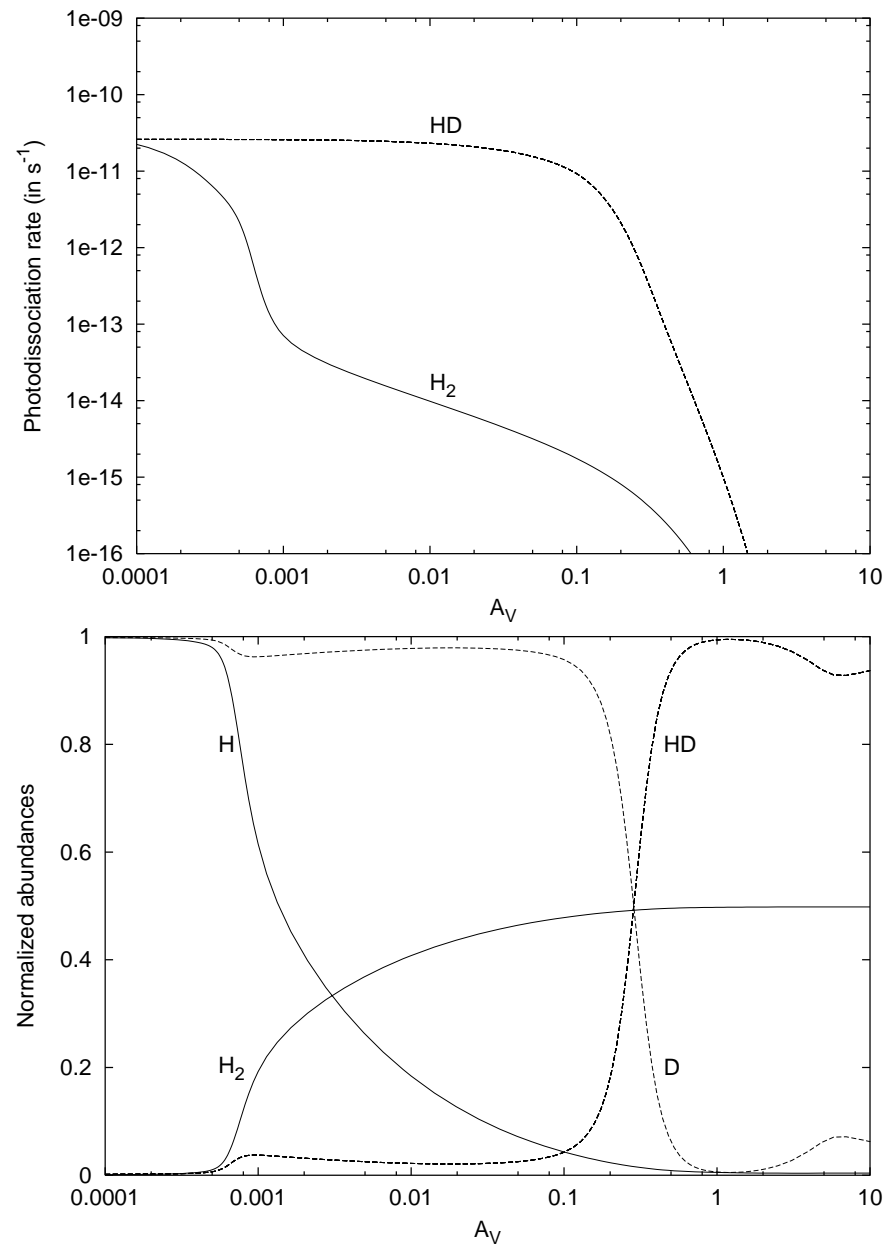

Fig. 1. Transition $\mathrm{H} / \mathrm{H}_{2}$ and $\mathrm{D} / \mathrm{HD}$ in the standard models. On top panel, photodissociation rates in $\mathrm{s}^{-1}$ of $\mathrm{H}_{2}$ and $\mathrm{HD}$ versus $A_{V}$. On bottom panel, normalized abundances of $\mathrm{H}, \mathrm{H}_{2}, \mathrm{D}$ and $\mathrm{HD}$ as a function of $A_{V}$.

The contributions of the different chemical processes related to the formation and the destruction of HD are displayed in Fig. 3. In this model, formation of HD on dust takes place as long as no molecular hydrogen is present. Once $\mathrm{H}_{2}$ is available, $\mathrm{HD}$ is formed through the reaction between $\mathrm{D}^{+}$and $\mathrm{H}_{2}$ (reaction 9). Photodissociation of HD dominates over other chemical destruction processes up to a visual extinction of about 1 magnitude.

There is a significant range of visual extinctions where deuterium is mainly atomic whereas hydrogen is predominantly molecular (cf. Fig. 1). The molecular fraction derived from the observations is defined as:

$f=\frac{2 N\left(\mathrm{H}_{2}\right)}{N(\mathrm{H})+2 N\left(\mathrm{H}_{2}\right)}$

where $N(\mathrm{H})$ and $N\left(\mathrm{H}_{2}\right)$ are the atomic and molecular column densities of hydrogen. Figure 4 displays the ratio of the atomic column densities, $N(\mathrm{D}) / N(\mathrm{H})$, and of the molecular column densities, $N(\mathrm{HD}) / 2 N\left(\mathrm{H}_{2}\right)$, as a function of the molecular fraction for the same standard model. We note that the atomic ratio, $N(\mathrm{D}) / N(\mathrm{H})$, is larger than the elemental $\mathrm{D} / \mathrm{H}$ ratio as soon as HD is present. The corresponding enhancement can reach about one order of magnitude for molecular fractions larger

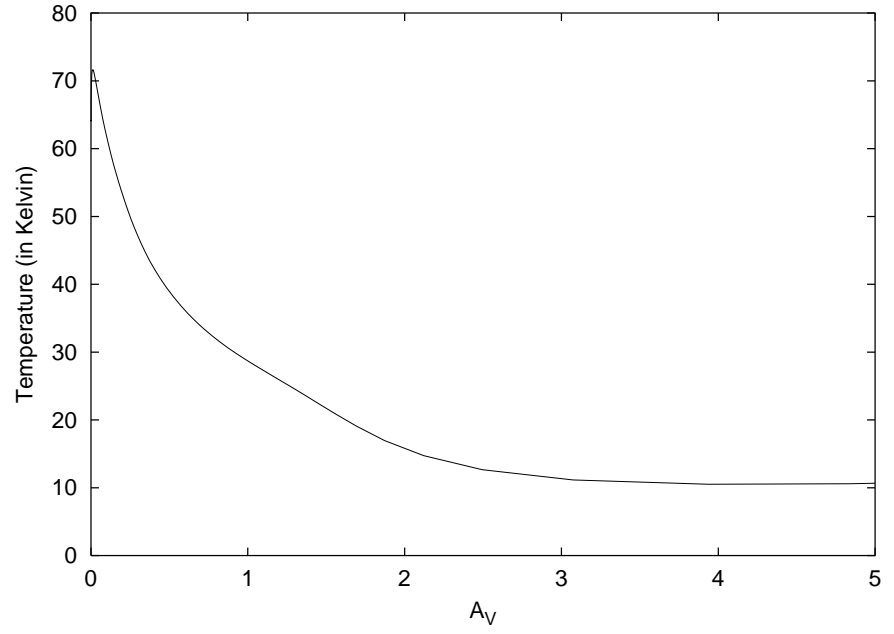

Fig. 2. Profile of the gas temperature as a function of $A_{V}$ in the standard model.

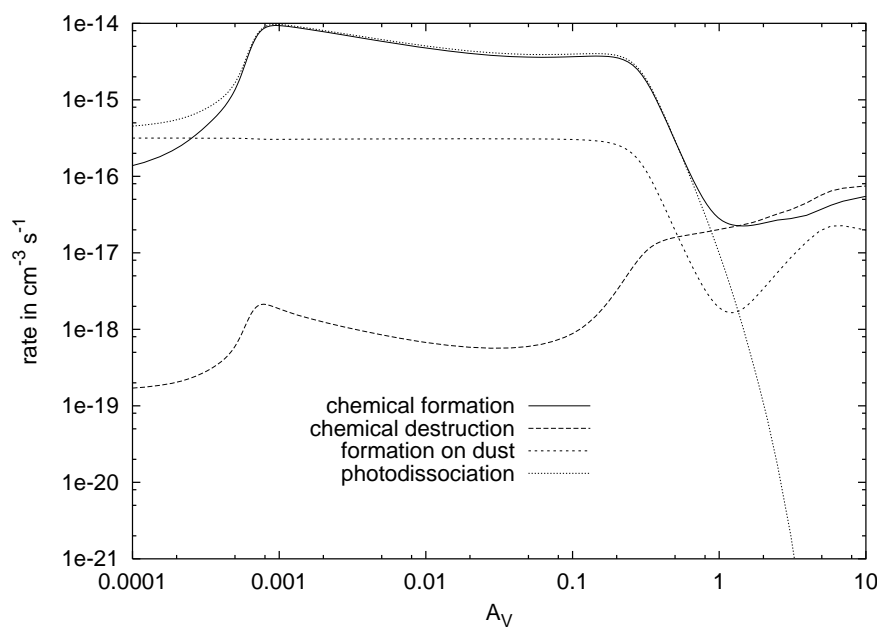

Fig. 3. Formation and destruction rates of $\mathrm{HD}$ in $\mathrm{cm}^{-3} \mathrm{~s}^{-1}$ versus visual extinction in the case of the standard model.

than 0.3. Such a possibility was already pointed out by Black \& Dalgarno (1973). On the other hand, the molecular ratio, $N(\mathrm{HD}) / 2 N\left(\mathrm{H}_{2}\right)$, is lower than the elemental ratio as long as deuterium is not totally in molecular form contrary to hydrogen. So, the derivation of the elemental value of deuterium from either atomic or molecular observations should be taken with care.

\subsubsection{Excitation of HD}

We display in Fig. 5 the column densities of the three first rotational levels of $\mathrm{HD}(J=0,1$ and 2$)$. The column density of HD $J=0$ increases as a function of the visual extinction, whereas those of $J=1$ and $J=2$ levels become stationary from $A_{V}$ about 0.5 , where the temperature is lower than $40 \mathrm{~K}$ (cf. Fig. 2). We find that the corresponding populations are sub-thermal as the density is below the critical density (cf. Tables 4 and 5). The column densities at $A_{V}=1$, are respectively $2.8 \times 10^{16} \mathrm{~cm}^{-2}$, $3.0 \times 10^{14} \mathrm{~cm}^{-2}$ and $6.9 \times 10^{10} \mathrm{~cm}^{-2}$, for $J=0,1$ and 2 . 


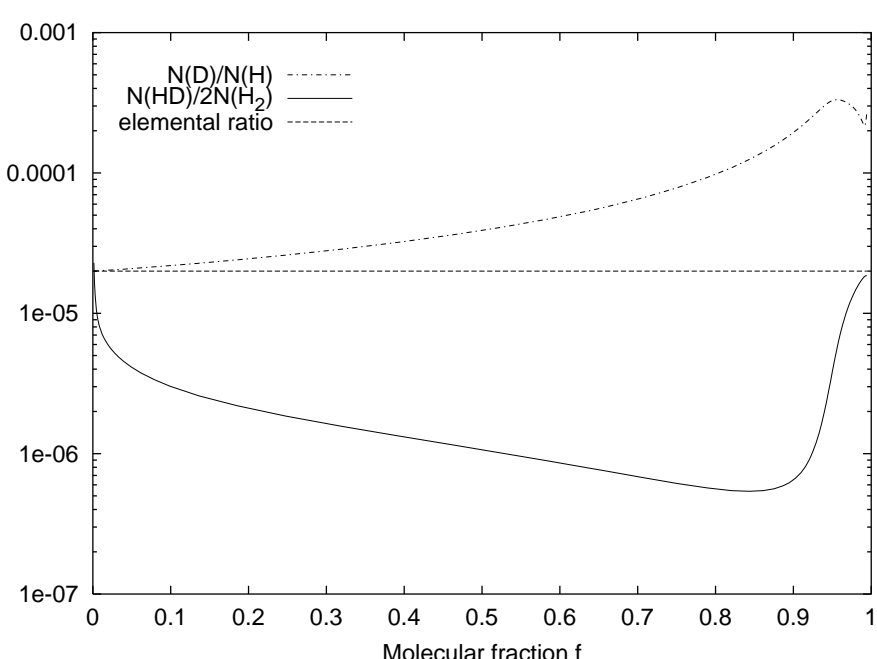

Fig. 4. Ratio of column densities $N(\mathrm{D}) / N(\mathrm{H})$ and $N(\mathrm{HD}) / 2 N\left(\mathrm{H}_{2}\right)$ as a function of the molecular fraction for the standard model. The horizontal line displays the $\mathrm{D} / \mathrm{H}$ elemental ratio adopted in the model: $2 \times 10^{-5}$.

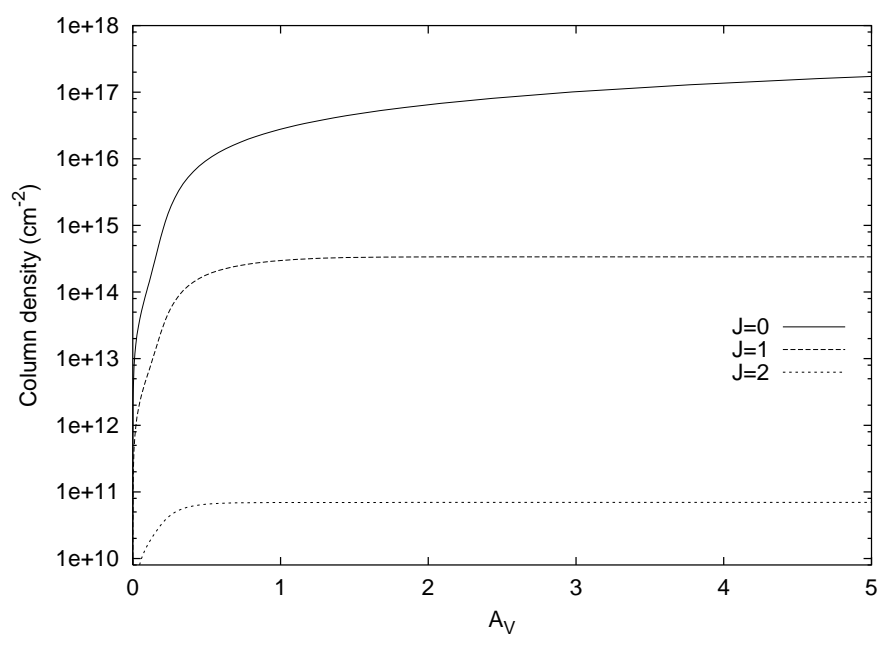

Fig. 5. Column densities of HD in the fundamental and first rotational levels, $J=0, J=1$ and $J=2$ in function of $A_{V}$ in the case of the standard model.

\subsection{Dependence on the cloud parameters}

\subsubsection{Role of the cosmic ray ionization rate}

Hartquist et al. (1978) have shown that the cosmic ionization rate could be derived from the observations of $\mathrm{OH}$ and HD. Approximate formulae of the steady state abundances of HD and $\mathrm{OH}$ have been obtained by Federman et al. (1996), for a molecular fraction of $1 / 3$, i.e. when $n(\mathrm{H}) / n\left(\mathrm{H}_{2}\right) \simeq 2$.

The first step of the chemistry of $\mathrm{OH}$ and HD involves the cosmic ray ionization of atomic hydrogen:

$\mathrm{H}+$ c.r. $\stackrel{k_{\zeta}}{\longrightarrow} \mathrm{H}^{+}+\mathrm{e}^{-}$

where c.r. stands for cosmic ray. This reaction is followed by slightly endothermic charge exchange reactions between $\mathrm{H}^{+}$ and $\mathrm{D}(\Delta H=43 \mathrm{~K})$ or $\mathrm{O}(\Delta H=227 \mathrm{~K})$ producing $\mathrm{D}^{+}$and $\mathrm{O}^{+}$. Then, reactions of $\mathrm{D}^{+}$and $\mathrm{O}^{+}$with $\mathrm{H}_{2}$ lead respectively to the formation of HD (Eq. (9)) and to a sequence of reactions leading to the formation of $\mathrm{OH}$. As long as photodissociation dominates the destruction of HD, the steady state abundance of HD can be expressed by:

$$
\begin{aligned}
n(\mathrm{HD}) & \simeq \frac{k_{1} k_{3} n\left(\mathrm{H}_{2}\right) n(\mathrm{D})}{\mathcal{P}_{\tau}(\mathrm{HD})} \\
& \times \frac{k_{\zeta} n(\mathrm{H})+k_{\zeta}^{\prime \prime} n\left(\mathrm{H}_{2}\right)+k_{4} n\left(\mathrm{H}_{2}\right) n\left(\mathrm{H}_{\mathrm{e}}^{+}\right)}{\left[k_{3}^{\prime} n(\mathrm{H})+k_{1} n\left(\mathrm{H}_{2}\right)\right]\left[k_{5} n(\mathrm{O})+\alpha\left(\mathrm{H}^{+}\right) n\left(\mathrm{e}^{-}\right)\right]}
\end{aligned}
$$

with

$n\left(\mathrm{H}_{\mathrm{e}}^{+}\right) \simeq \frac{k_{\zeta}^{\prime} n\left(\mathrm{H}_{\mathrm{e}}\right)}{\alpha\left(\mathrm{H}_{\mathrm{e}}^{+}\right) n\left(\mathrm{e}^{-}\right)+k_{4} n\left(\mathrm{H}_{2}\right)}$

where $\mathcal{P}_{\tau}(\mathrm{HD})$ is the photodissociation rate $\left(\right.$ in $\left.^{-1}\right)$ of $\mathrm{HD}$ at the optical depth $\tau$. The corresponding reaction rate coefficients, $k_{i}$, are given in Table 6.

As for $\mathrm{OH}$, its steady state abundance can be written:

$$
\begin{gathered}
n(\mathrm{OH}) \simeq \frac{\alpha_{2}+\alpha_{3}}{\alpha_{1}+\alpha_{2}+\alpha_{3}} \frac{k_{5} n(\mathrm{O})}{\mathcal{P}_{\tau}(\mathrm{OH})+k_{6} n\left(\mathrm{C}^{+}\right)} \\
\times \frac{k_{\zeta} n(\mathrm{H})+k_{\zeta}^{\prime \prime} n\left(\mathrm{H}_{2}\right)+k_{4} n\left(\mathrm{H}_{2}\right) n\left(\mathrm{H}_{\mathrm{e}}^{+}\right)}{k_{5} n(\mathrm{O})+\alpha\left(\mathrm{H}^{+}\right) n\left(\mathrm{e}^{-}\right)} \\
\text {with } \quad n\left(\mathrm{C}^{+}\right)=\frac{\mathcal{P}_{\tau}(\mathrm{C}) n(\mathrm{C})}{\alpha\left(\mathrm{C}^{+}\right) n\left(\mathrm{e}^{-}\right)} .
\end{gathered}
$$

In this equation, $\mathcal{P}_{\tau}(\mathrm{C})$ and $\mathcal{P}_{\tau}(\mathrm{OH})$ are respectively the photoionization rate of carbon and the photodissociation rate of $\mathrm{OH}$ at the optical depth $\tau$.

Our expressions differ slightly from those given by Federman et al. (1996). In particular, they remain valid in the range where $\mathrm{H}$ is mainly molecular whereas HD is still photodissociated efficiently. This can be seen in Eqs. (18) and (19), where the numerator of the second fraction expresses the production of $\mathrm{H}^{+}$not only through cosmic ray ionization of atomic hydrogen (sole case considered by Federman et al.) but also via cosmic ray ionization of molecular hydrogen and via $\mathrm{He}^{+}+\mathrm{H}_{2}$. So, the abundances of HD and $\mathrm{OH}$ are proportional to the cosmic ionization rate, $\zeta$.

Once chemical destruction of $\mathrm{HD}$ by $\mathrm{H}_{3}^{+}$is dominant, the proportionality to $\zeta$ is no more fulfilled since the abundance of $\mathrm{H}_{3}^{+}$is itself proportional to the cosmic ray ionization rate.

This point is illustrated in Fig. 6 where we display the column densities of HD and $\mathrm{OH}$ divided by $\zeta$, computed for models of constant density $\left(n_{\mathrm{H}}=500 \mathrm{~cm}^{-3}\right)$ and different values of $\zeta$ as a function of the visual extinction. The ratios are independent of $\zeta$ in a range of $A_{V}$ between 0.001 and 0.1 for HD and 0.001 and 1 for $\mathrm{OH}$.

\subsubsection{Dependence on the density and radiation field}

\section{D/HD transition}

Figure 7 displays the normalized abundances of D and HD calculated in models with densities between 100 and $10^{5} \mathrm{~cm}^{-3}$ and $\chi=1$. The respective atomic and molecular abundances of deuterium at the edge of the cloud are directly obtained from the balance between photodissociation and formation on dust.

$\left.\frac{n(\mathrm{HD})}{n(\mathrm{D})}\right|_{\text {edge }}=\frac{\mathcal{F} \mathcal{R}(\mathrm{HD})}{\mathcal{P}_{0}(\mathrm{HD})}=\frac{6.3 \times 10^{-17} n_{\mathrm{H}}}{2.6 \times 10^{-11}}=2.4 \times 10^{-6} n_{\mathrm{H}}$ 
Table 6. Reaction rate coefficients.

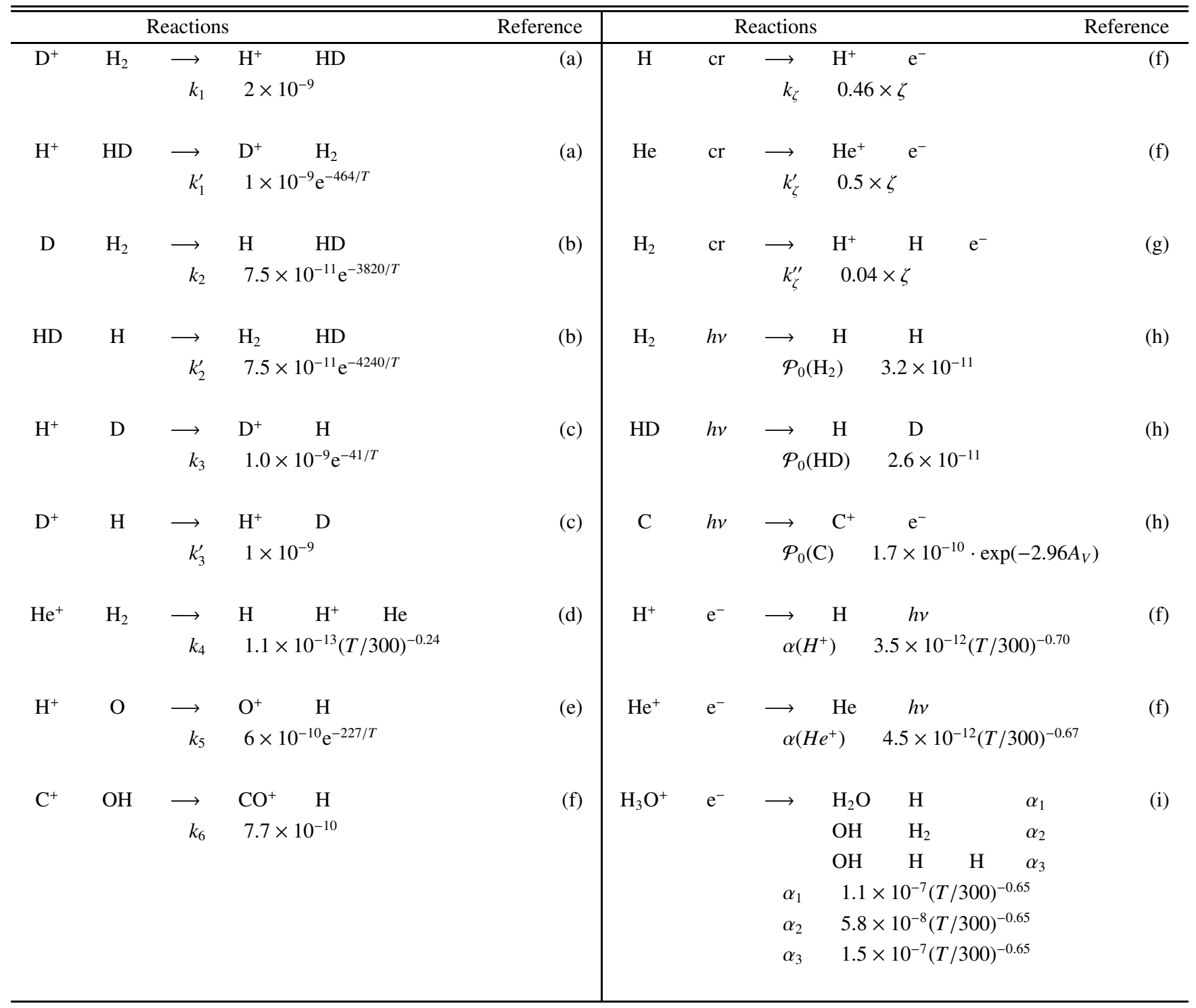

All rates are in $\mathrm{cm}^{3} \mathrm{~s}^{-1}$ excepted those of photodissociation, photoionization and ionization by cosmic rays wich are in $\mathrm{s}^{-1}$. References: (a) Smith, D., Adams, N. G., \& Alge, E. 1982, ApJ, 263, 123. (b) Stancil, P. C., Lepp, S., \& Dalgarno, A. 1998, ApJ, 509, 1. (c) Watson, W. D. 1976, Rev. Mod. Phys., 48, 513. (d) Böringer, H., \& Arnold, F. 1986, J. Chem. Phys., 84, 1459. (e) Federer, W., et al. 1984, Phys. Rev. Lett., 52, 2084. (f) Prasad, S. S., \& Huntress, W. T. 1980, ApJS, 43, 1. (g) Private communication from Alex Dalgarno. (h) Obtained from our numerical model computing the photodissociation of the chemical species. (i) Vejby-Chriestensen, J., et al. 1997, ApJ, 483, 531.

where $\mathcal{P}_{0}(\mathrm{HD})$ is the photodissociation rate of $\mathrm{HD}$ in $\mathrm{s}^{-1}$ at the edge of the cloud.

We introduce $A_{V_{\mathrm{D} / \mathrm{HD}}}$, the value of the visual extinction where $n(\mathrm{D})=n(\mathrm{HD})=\xi_{\mathrm{D}} \cdot n_{\mathrm{H}} / 2$ with $\xi_{\mathrm{D}}$, the elemental abundance of deuterium relative to $\mathrm{H}$.

In the case where $\mathrm{HD}$ is formed by $\mathrm{D}^{+}+\mathrm{H}_{2}$, neglecting the contribution of atomic hydrogen and assuming that $\mathrm{C}$ is the main source of electrons, we obtain a relation between the photodissociation rate at the D/HD transition, the different rate coefficients, and the elemental abundances relative to $\mathrm{H}$ of $\mathrm{He}\left(\xi_{\mathrm{He}}\right), \mathrm{C}\left(\xi_{\mathrm{C}}\right)$ and $\mathrm{O}\left(\xi_{\mathrm{O}}\right)$.

$$
\left.P_{\tau}(\mathrm{HD})\right|_{\text {transition }}=\frac{k_{3}\left[k_{\zeta}^{\prime} \xi_{\mathrm{He}}+k_{\zeta}^{\prime \prime} / 2\right]}{\left[\alpha\left(H^{+}\right) \xi_{\mathrm{C}}+k_{5} \xi_{\mathrm{O}}\right]} .
$$

The photodissociation rate (in $\mathrm{s}^{-1}$ ) at the optical depth $\tau$ can be expressed as:

$\mathcal{P}_{\tau}(\mathrm{HD})=\chi \mathcal{P}_{0}(\mathrm{HD}) f(N(\mathrm{HD})) \mathrm{e}^{-\beta A_{V}} \quad\left(\mathrm{~s}^{-1}\right)$

where $\exp \left(-\beta A_{V}\right)$ corresponds to the continuum absorption by grains and $f(N(\mathrm{HD}))$ represents the self-shielding function and depends only on $N(\mathrm{HD})$. We ignore the possible overlap with $\mathrm{H}_{2}$ lines which is a complex function of the column density of $\mathrm{H}_{2}$.

The substitution of this expression in Eq. (20) implies at first sight that $A_{V_{\mathrm{D}} / \mathrm{HD}}$ should not depend on the density. This first guess should be tempered since the thermal profile is density dependent and $A_{V_{\mathrm{D} / \mathrm{HD}}}$ is a sensitive function of the kinetic temperature via the rate coefficients. This can be seen in Fig. 8 

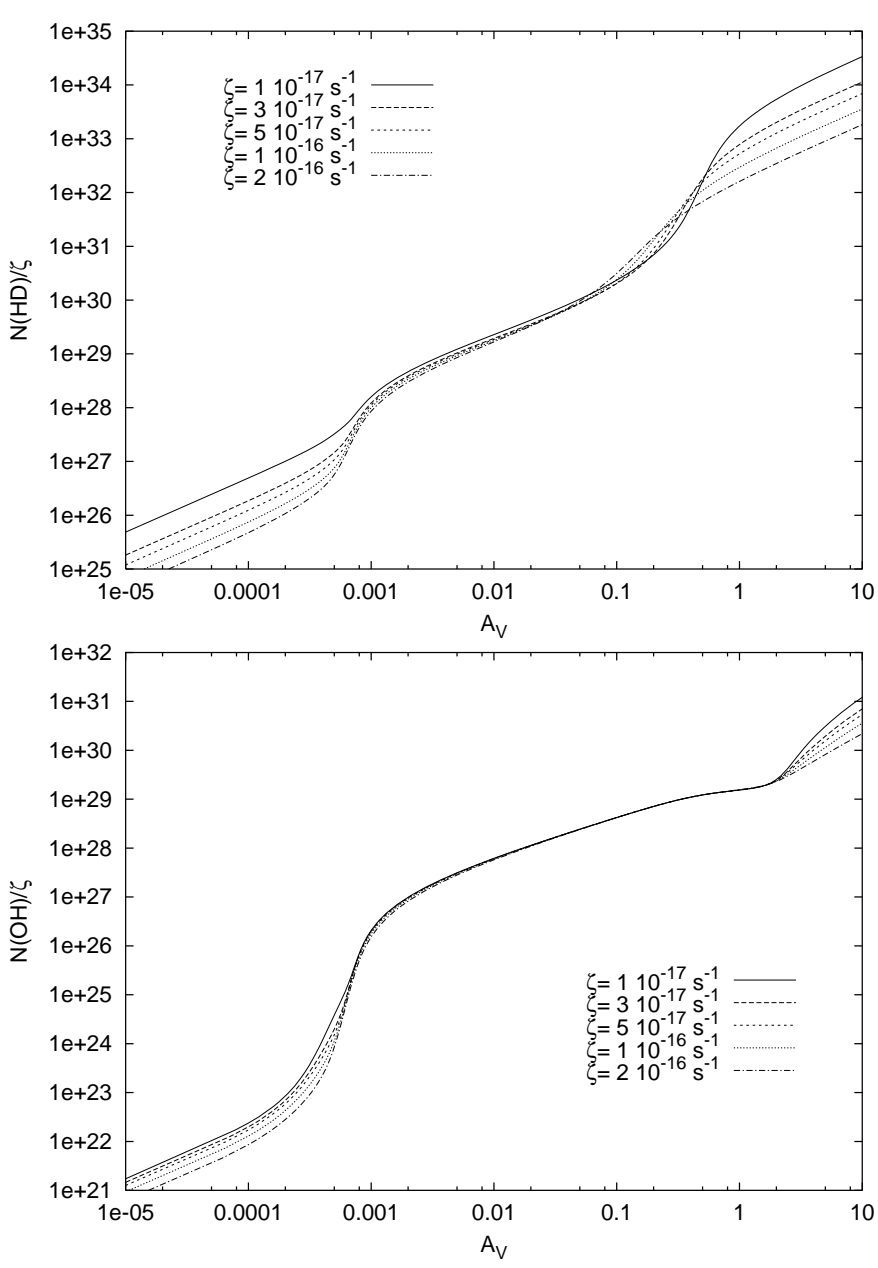

Fig. 6. $N(\mathrm{HD}) / \zeta$ and $N(\mathrm{OH}) / \zeta$ in function of the visual extinction for a model of density $n_{\mathrm{H}}=500 \mathrm{~cm}^{-3}$ and $\chi=1$, in a log-log diagram.

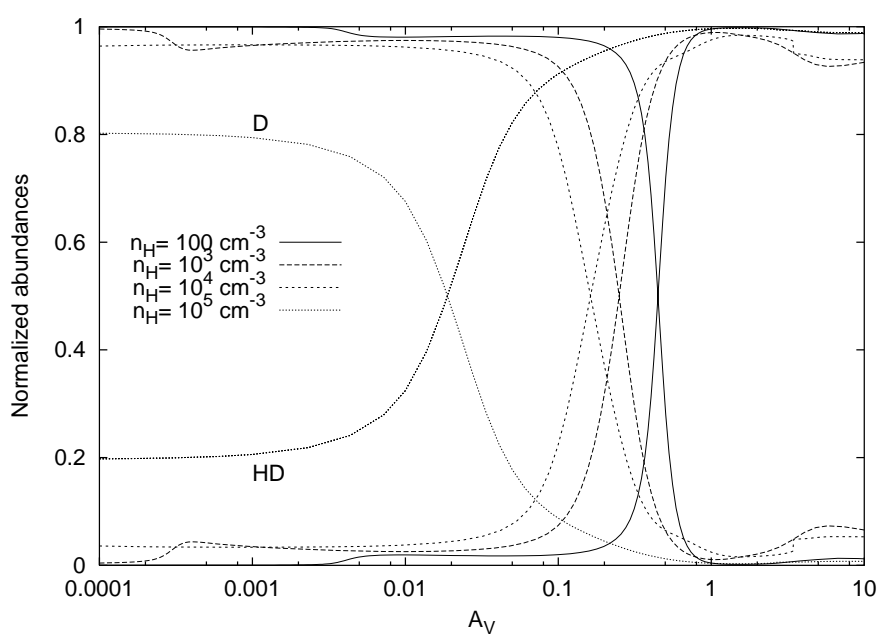

Fig. 7. D/HD transition for models with densities between 100 and $10^{5} \mathrm{~cm}^{-3}$ and $\chi=1$.

which displays $A_{V_{\mathrm{D} / \mathrm{HD}}}$ as well as the temperature at the $\mathrm{D} / \mathrm{HD}$ transition, $T_{\mathrm{D} / \mathrm{HD}}$, for different densities and for $\chi=1$. This behaviour is different from the $\mathrm{H} / \mathrm{H}_{2}$ transition where the formation rate on dust is proportional to the square of the density. The dependence of $A_{V_{\mathrm{D} / \mathrm{HD}}}$ on $\chi$ is $\ln (\chi)$.

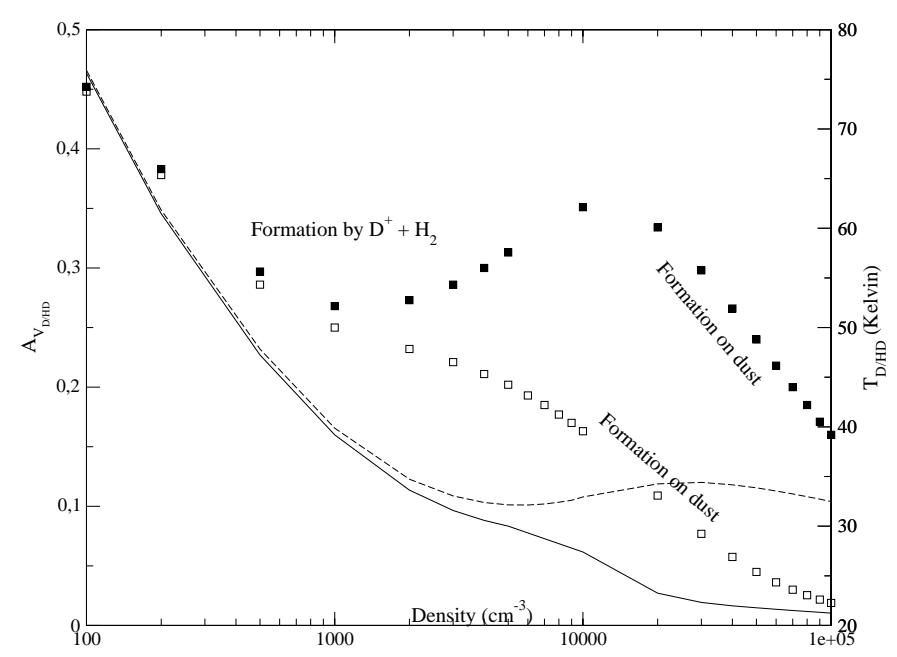

Fig. 8. Position of the transition D/HD, $A_{V_{\mathrm{D} / \mathrm{HD}}}$ (squares) and temperature of the gas (lines) at the transition as a function of the density. Blank squares and dashed line correspond to the standard formation rate of HD on dust $\left(\mathcal{F} \mathcal{R}(\mathrm{HD})=6.3 \times 10^{-17} \times n_{\mathrm{H}}\right)$. Black squares and full line correspond to $\mathcal{F} \mathcal{R}(\mathrm{HD})=6.3 \times 10^{-18} \times n_{\mathrm{H}}$. For all models, $\chi=1$.

As described in Sect. 2.3, at densities higher than $5000 \mathrm{~cm}^{-3}$, the formation of HD occurs principally on dust. In this case, at the $\mathrm{D} / \mathrm{HD}$ transition:

$$
\left.P_{\tau}(\mathrm{HD})\right|_{\text {transition }}=k_{\mathrm{ads}}=6.3 \times 10^{-17} n_{\mathrm{H}} \text {. }
$$

With the photodissociation rate expression (Eq. (21)), we derive that $A_{V_{\mathrm{D} / \mathrm{HD}}}$ is now a function of $\ln \left(\chi / n_{\mathrm{H}}\right)$. This logarithmic behaviour can be seen in Fig. 8 for densities higher than $5000 \mathrm{~cm}^{-3}$ when standard formation rate of HD on dust is assumed.

\section{Dependence on the formation rate of $H D$ on dust}

The formation rate of HD on dust is not well known. We discuss the significance of the mechanism introducing a formation rate with a sticking factor 10 times less than the standard hypothesis. Considering the full squares symbols in Fig. 8 two different regimes are still found for $A_{V_{\mathrm{D} / \mathrm{HD}}}$.

As expected, the regime corresponding to formation on dust is taking place at larger densities $\left(n_{\mathrm{H}} \geq 20000 \mathrm{~cm}^{-3}\right)$ than with the standard rate.

We have compared the computed column densities of HD at a visual extinction of 1 , obtained using the two different HD formation rates on dust. The differences in the column densities are smaller than $10 \%$ for densities between $10^{3}$ and $10^{4} \mathrm{~cm}^{-3}$ and of the order of $30 \%$ for higher densities.

\section{Dependence on the intensity of the incident radiation field}

Figure 9 displays $A_{V_{\mathrm{D} / \mathrm{HD}}}$ and the temperature at the transition as a function of the scaling factor $\chi$ of the ISRF for models with densities 500 and $10^{5} \mathrm{~cm}^{-3}$. When $n_{\mathrm{H}}=500 \mathrm{~cm}^{-3}$, the formation of HD occurs always through the gas phase reaction between $\mathrm{D}^{+}$and $\mathrm{H}_{2}$ and the variation of $A_{V_{\mathrm{D} / \mathrm{HD}}}$ with $\chi$ is almost logarithmic as predicted by Eq. (20). For $n_{\mathrm{H}}=10^{5} \mathrm{~cm}^{-3}$ and $\chi$ lower than 2600 the formation of HD occurs on dust. For larger 


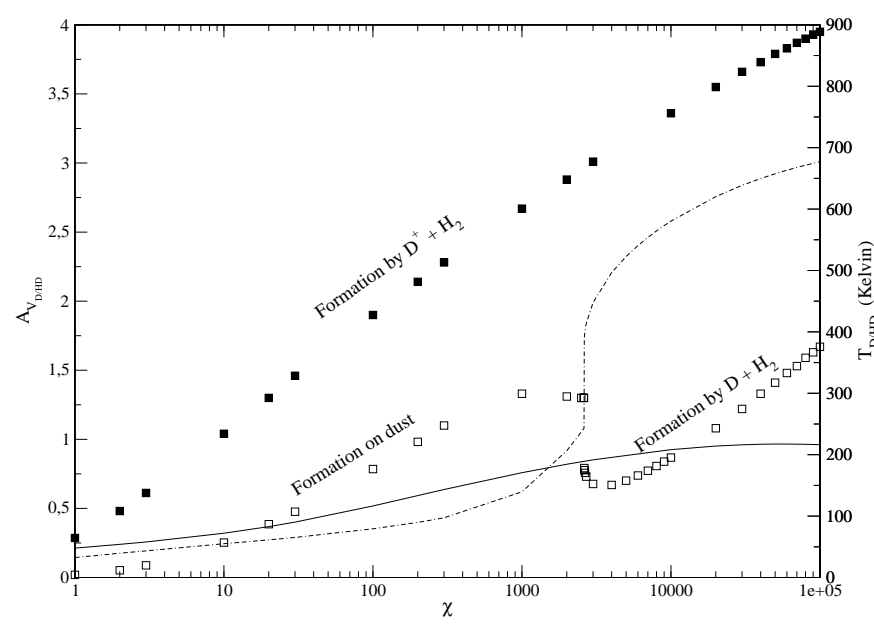

Fig. 9. Position of the transition $\mathrm{D} / \mathrm{HD}, A_{V_{\mathrm{D} / \mathrm{HD}}}$ (squares) and temperature (lines) at the transition versus $\chi$. Black squares and full line correspond to models of density $500 \mathrm{~cm}^{-3}$ while blank squares and dashed line correspond to models of density $10^{5} \mathrm{~cm}^{-3}$.

values of $\chi$, the temperature may reach several hundred Kelvin allowing the neutral-neutral reaction $\mathrm{D}+\mathrm{H}_{2}$ to become dominant. The high efficiency of this process moves the position of the transition D/HD closer to the edge of the cloud, producing a discontinuity as shown on the figure. Under these conditions, $A_{V_{\mathrm{D} / \mathrm{HD}}}$ is given by:

$\mathcal{P}_{\tau}(\mathrm{HD})+k_{2}^{\prime} n(\mathrm{H})=k_{2} n\left(\mathrm{H}_{2}\right) \simeq \frac{k_{2}}{2} n_{\mathrm{H}}$

where the reverse reaction may not be negligible. Once more, a logarithmic dependence on $\chi$ as $\ln \left(\chi / n_{\mathrm{H}}\right)$ is obtained for $A_{V_{\mathrm{D} / \mathrm{HD}}}$, as displayed in the figure.

\subsection{Validity of the equilibrium model}

Our model is steady state. A characteristic timescale can be derived from the destruction processes of HD.

$t(\mathrm{HD})_{\text {dest. }}=\frac{n(\mathrm{HD})}{\text { destruction rate in } \mathrm{cm}^{-3} \mathrm{~s}^{-1}}$.

While photodissociation is the main process of destruction of $\mathrm{HD}$, the characteristic timescale is equal to the inverse of the photodissociation rate (in $\mathrm{s}^{-1}$ ). Its value is $1000 / \chi$ years at the edge of the cloud and it increases when self-shielding and dust absorption become significant. In the absence of photoprocesses, HD is principally destroyed by $\mathrm{H}_{3}^{+}$, via reaction 14 . As $\mathrm{H}_{3}^{+}$is directly formed via the reaction between $\mathrm{H}_{2}^{+}$and $\mathrm{H}_{2}$ and destroyed by reactions with $\mathrm{O}, \mathrm{CO}$ and dissociative recombination, the chemical time associated to HD is inversely proportional to $n_{H}$. As long as this chemical timescale is smaller than a dynamic time scale, for example the free fall time, the steady state approximation is adequate. This condition is reasonably fulfilled in the translucent part of the model. Time dependent models such as those presented by Lee et al. (1996) for $\mathrm{H}_{2}$ and $\mathrm{CO}$ may be more appropriate in the more obscured regions.

\section{Comparison with observations}

\subsection{UV absorption of $H D$}

The present model enables interpretation of the available and forthcoming observations of UV absorption transitions of $\mathrm{H}$, $\mathrm{D}, \mathrm{H}_{2}, \mathrm{HD}$, and other species towards diffuse and translucent clouds. At present, the high sensitivity of FUSE allows to extend the sample of diffuse clouds in front of bright stars obtained by Copernicus and IUE satellites towards lines of sight involving more reddened stars. However, derivation of the column density of HD is subject to large uncertainties since HD lines are partially saturated and several components may be present on the lines of sight. We have shown in Sect. 3.1, that the elemental deuterium to hydrogen ratio is not simply given by $N(\mathrm{HD}) / 2 N\left(\mathrm{H}_{2}\right)$. Table 7 displays $N(\mathrm{D}) / N(\mathrm{H})$ and $N(\mathrm{HD}) / 2 N\left(\mathrm{H}_{2}\right)$ for different values of the molecular fraction deduced from our standard model $\left(n_{\mathrm{H}}=500 \mathrm{~cm}^{-3}, \chi=1\right)$. Results are given for elemental $\mathrm{D} / \mathrm{H}$ ratios of $1 \times 10^{-5}$ and of $2 \times 10^{-5}$. The values of both $N(\mathrm{D}) / N(\mathrm{H})$ and $N(\mathrm{HD}) / 2 N\left(\mathrm{H}_{2}\right)$ are proportional to the elemental ratio $\xi_{\mathrm{D}}$. As expected they bracket the actual value of $\xi_{\mathrm{D}}$.

For high $z$ objects, UV absorption transitions are shifted to the visible. The first detection of $\mathrm{HD}(N(\mathrm{HD})=$ $1-4 \times 10^{14} \mathrm{~cm}^{-2}$ ) in front of a quasar (PKS 1232+082) is reported by Varshalovich et al. (2001). On the other hand, Petitjean et al. (2000) find a column density of $\mathrm{H}_{2}$ of $6 \times 10^{16} \mathrm{~cm}^{-2}$ and a molecular fraction of $1.5 \times 10^{-4}$ towards the same line of sight. We can deduce from these values $\mathrm{N}(\mathrm{HD}) / 2 \mathrm{~N}(\mathrm{H} 2)$ of $8.3 \times 10^{-4}-3.3 \times 10^{-3}$. This surprising result may come from the specific physical conditions present in extragalactic environment.

\subsection{Infrared intensities of HD rotational transitions}

Our model of the HD excitation allows to derive the intensities of the pure rotational transitions of HD. As the transitions probabilities are low, the intensities $I_{J, J-1}$ are directly obtained from an optically thin approximation:

$I_{J, J-1}=\frac{N(J)}{4 \pi} A_{J, J-1} E_{J, J-1}$

where $N(J)$ is the column density of the upper level of the transition, $A_{J, J-1}$, the Einstein emission probabilities and $E_{J, J-1}$ the energy of the transition. We display the corresponding values in Tables 8 and 9 for the transitions at $112 \mu \mathrm{m}(J=1 \rightarrow 0)$ and $56 \mu \mathrm{m}(J=2 \rightarrow 1)$. Results are expressed in erg s $\mathrm{s}^{-1} \mathrm{~cm}^{-2} \mathrm{sr}^{-1}$ and have been calculated for a total visual extinction of 10 magnitudes and densities between 100 and $10^{5} \mathrm{~cm}^{-3}$ and $\chi$ between 1 and $10^{5}$. The kinetic temperature at the emission maximum of the transition and the corresponding visual extinction are also displayed in Tables 8 and 9 for each model in order to better explain the intensity values. The models sample a large range of physical conditions.

The magnitudes of the intensities of both infrared transitions are principally driven by the magnitude of the radiation field. The position of the local emissivity maximum is shifted towards larger values of the visual extinction as $\chi$ increases and the temperature increases as well. The two transitions are not 
Table 7. Column densities ratios in function of $f$.

\begin{tabular}{ccc}
\hline \hline$f$ & $N(\mathrm{D}) / N(\mathrm{H})^{(a)}$ & $N(\mathrm{HD}) /\left(2 \times N\left(\mathrm{H}_{2}\right)\right)^{(a)}$ \\
\hline 0.1 & $2.2(-5)$ & $3.0(-6)$ \\
& $1.1(-5)$ & $1.5(-6)$ \\
0.2 & $2.5(-5)$ & $2.0(-6)$ \\
& $1.2(-5)$ & $1.1(-6)$ \\
0.3 & $2.8(-5)$ & $1.6(-6)$ \\
& $1.4(-5)$ & $7.8(-7)$ \\
0.4 & $3.2(-5)$ & $1.3(-6)$ \\
& $1.6(-5)$ & $6.7(-7)$ \\
0.5 & $4.1(-5)$ & $1.0(-6)$ \\
& $2.0(-5)$ & $5.1(-7)$ \\
0.6 & $5.0(-5)$ & $8.2(-7)$ \\
& $2.4(-5)$ & $4.0(-7)$ \\
0.7 & $6.7(-5)$ & $6.7(-7)$ \\
& $3.3(-5)$ & $3.4(-7)$ \\
0.8 & $9.6(-5)$ & $5.5(-7)$ \\
& $4.8(-5)$ & $2.8(-7)$ \\
0.9 & $2.0(-4)$ & $6.7(-7)$ \\
& $1.0(-4)$ & $3.2(-7)$ \\
0.99 & $2.7(-4)$ & $1.9(-5)$ \\
& $1.4(-4)$ & $9.3(-6)$ \\
\hline
\end{tabular}

(a) Values have been obtained in the case of the standard model $\left(n_{\mathrm{H}}=\right.$ $500 \mathrm{~cm}^{-3}, \chi=1$ ). For each value of $f$ (molecular fraction), the first line corresponds to $\xi_{\mathrm{D}}=2 \times 10^{-5}$ and the second one to $\xi_{\mathrm{D}}=1 \times 10^{-5}$. Numbers in parentheses refer to power of ten.

exactly peaking at the same visual extinctions since the $56 \mu \mathrm{m}$ transition corresponds to a higher excitation. The intensity of the $112 \mu \mathrm{m}$ transition reported by Wright et al. (1999) towards the Orion Bar is $(8.7 \pm 1.5) \times 10^{-6} \mathrm{erg} \mathrm{s}^{-1} \mathrm{~cm}^{-2} \mathrm{sr}^{-1}$. As the radiation field in this environment is estimated to be close to $4.4 \times 10^{4}$ times the ISRF (Parmar et al. 1991; Hogerheijde et al. 1995 ) and the mean density value is $2.5 \times 10^{5} \mathrm{~cm}^{-3}$ (Jansen et al. 1995), the observed value is compatible with our results. However, the knowledge of the actual geometry is critical to make quantitative predictions. The ratio of the emissivities of the $112 \mu \mathrm{m}$ and $56 \mu \mathrm{m}$ transitions is a very sensitive function of both $n_{H}$ and $\chi$. However, the $56 \mu \mathrm{m}$ has a lower intensity except for very high density and very large radiation field conditions. It has not been detected with ISO. The future HERSCHEL mission will allow to search for the $112 \mu \mathrm{m}$ transition at a much higher sensitivity than ISO with the PACS instrument. A sensitivity of some $10^{-14} \mathrm{erg} \mathrm{s}^{-1} \mathrm{~cm}^{-2}$ in a field of view of $47^{\prime \prime} \times$ $47 "$ is expected. The $56 \mu \mathrm{m}$ transition is unfortunately beyond the reserved wavelength range of HERSCHEL.

\section{Conclusion and perspectives}

We have presented PDR models where chemistry and rotational excitation of HD is explicitly introduced with the most recent molecular data. Thermal balance is solved in parallel to chemical equilibrium. As for $\mathrm{H}_{2}$, photodissociation in the discrete UV transitions of Lyman and Werner electronic bands con-
Table 8. Intensities at $112 \mu \mathrm{m}$.

\begin{tabular}{|c|c|c|c|c|c|}
\hline & \multicolumn{5}{|c|}{$n_{\mathrm{H}}\left(\mathrm{cm}^{-3}\right)$} \\
\hline & 100 & 1000 & $1 \times 10^{4}$ & $1 \times 10^{5}$ & $1 \times 10^{6}$ \\
\hline \multicolumn{6}{|l|}{$\chi$} \\
\hline \multirow[t]{3}{*}{1} & $2.5(-8)$ & $2.3(-8)$ & $2.3(-8)$ & $1.2(-8)$ & $2.2(-9)$ \\
\hline & 78 & 40 & 104 & 143 & 155 \\
\hline & 0.45 & 0.26 & $1.8(-5)$ & $6.3(-7)$ & $1.0(-7)$ \\
\hline \multirow[t]{3}{*}{10} & $7.7(-8)$ & $1.1(-7)$ & $2.1(-7)$ & $2.5(-7)$ & $1.7(-7)$ \\
\hline & 111 & 59 & 47 & 50 & 46 \\
\hline & 1.3 & 1 & 0.78 & 0.35 & 0.07 \\
\hline \multirow[t]{3}{*}{100} & $1.9(-7)$ & $4.4(-7)$ & $7.8(-7)$ & $8.6(-7)$ & $8.7(-7)$ \\
\hline & 145 & 97 & 76 & 70 & 69 \\
\hline & 2 & 1.9 & 1.5 & 1 & 0.45 \\
\hline \multirow[t]{3}{*}{$10^{3}$} & $3.7(-7)$ & $1.2(-6)$ & $2.0(-6)$ & $2.2(-6)$ & $2.6(-6)$ \\
\hline & 176 & 156 & 124 & 108 & 111 \\
\hline & 2.8 & 2.8 & 2.35 & 1.7 & 1.0 \\
\hline \multirow[t]{3}{*}{$10^{4}$} & $5.6(-7)$ & $2.1(-6)$ & $4.2(-6)$ & $4.7(-6)$ & $4.9(-6)$ \\
\hline & 190 & 207 & 208 & 157 & 151 \\
\hline & 3.6 & 3.6 & 3.1 & 2.7 & 2.1 \\
\hline \multirow[t]{3}{*}{$10^{5}$} & $7.0(-7)$ & $3.1(-6)$ & $5.9(-6)$ & $5.7(-6)$ & $6.4(-6)$ \\
\hline & 189 & 238 & 248 & 165 & 154 \\
\hline & 4.8 & 4.4 & 4.3 & 4.1 & 3.5 \\
\hline
\end{tabular}

For each couple of density and $\chi$, the first line gives the intensity of the transition calculated for $A_{V}=10 \mathrm{in} \mathrm{erg} \mathrm{s}^{-1} \mathrm{~cm}^{-2} \mathrm{sr}^{-1}$. The second and third lines give the temperature and the visual extinction corresponding to the local emissivity maximum. Numbers in parentheses refer to power of ten.

trols the atomic to molecular deuterium transition. However, contrary to $\mathrm{H}_{2}$, gas phase chemical formation of HD is occurring via the $\mathrm{D}^{+}+\mathrm{H}_{2}$ reaction in low and medium density clouds. Competing formation mechanisms of HD are taking place for high density conditions. Formation on dust may be preponderant in cold dense regions whereas the neutral-neutral reaction between $\mathrm{D}$ and $\mathrm{H} 2$ takes place under high interstellar radiation field conditions where the temperature may reach several hundred Kelvin at the edge of the cloud. The D/HD transition occurs for larger visual extinctions than for $\mathrm{H} / \mathrm{H}_{2}$ since self-shielding effects are much less efficient due to the small elemental abundance of deuterium. We have also given the critical densities for different temperatures: at low densities $\left(n_{\mathrm{H}} \leq 1000 \mathrm{~cm}^{-3}\right)$, the excitation of the level $J=1$ of HD is sub-thermal whether the medium is atomic or molecular. The critical density values increase significantly when $J$ increases. This remains true for vibrational energy levels for which the Einstein emission coefficients are several $10^{-5} \mathrm{~s}^{-1}$ and the vibrational de-excitation coefficients are of the order of $10^{-15}$ at maximum. 
Table 9. Intensities at $56 \mu \mathrm{m}$.

\begin{tabular}{|c|c|c|c|c|c|}
\hline & \multicolumn{5}{|c|}{$n_{\mathrm{H}}\left(\mathrm{cm}^{-3}\right)$} \\
\hline & 100 & 1000 & $1 \times 10^{4}$ & $1 \times 10^{5}$ & $1 \times 10^{6}$ \\
\hline \multicolumn{6}{|l|}{$\chi$} \\
\hline \multirow[t]{3}{*}{1} & $2.9(-10)$ & $7.0(-11)$ & $1.0(-10)$ & $6.1(-11)$ & $7.2(-12)$ \\
\hline & 87 & 45 & 111 & 142 & 161 \\
\hline & 0.35 & 0.14 & $1.2(-5)$ & $6.3(-7)$ & $6.8(-8)$ \\
\hline \multirow[t]{3}{*}{10} & $2.4(-9)$ & $1.8(-9)$ & $8.5(-9)$ & $2.3(-8)$ & $7.6(-9)$ \\
\hline & 122 & 65 & 51 & 54 & 52 \\
\hline & 1.1 & 0.9 & 0.6 & 0.28 & 0.025 \\
\hline \multirow[t]{3}{*}{100} & $1.2(-8)$ & $3.5(-8)$ & $1.8(-7)$ & $3.3(-7)$ & $3.3(-7)$ \\
\hline & 152 & 109 & 84 & 75 & 75 \\
\hline & 1.9 & 1.8 & 1.4 & 0.9 & 0.36 \\
\hline \multirow[t]{3}{*}{$10^{3}$} & $3.2(-8)$ & $2.9(-7)$ & $1.7(-6)$ & $3.7(-6)$ & $6.3(-6)$ \\
\hline & 181 & 168 & 142 & 128 & 143 \\
\hline & 2.7 & 2.6 & 2.1 & 1.4 & 0.74 \\
\hline \multirow[t]{3}{*}{$10^{4}$} & $5.6(-8)$ & $8.9(-7)$ & $1.2(-5)$ & $2.7(-5)$ & $2.4(-5)$ \\
\hline & 190 & 215 & 272 & 390 & 404 \\
\hline & 3.6 & 3.4 & 2.6 & 1.6 & 1.2 \\
\hline \multirow[t]{3}{*}{$10^{5}$} & $6.7(-8)$ & $1.6(-6)$ & $2.5(-5)$ & $3.9(-5)$ & $3.4(-5)$ \\
\hline & 189 & 242 & 378 & 439 & 402 \\
\hline & 4.8 & 4.2 & 3.0 & 2.8 & 2.5 \\
\hline
\end{tabular}

For each couple of density and $\chi$, the first line gives the intensity of the transition calculated for $A_{V}=10$ in $\mathrm{erg} \mathrm{s}^{-1} \mathrm{~cm}^{-2} \mathrm{sr}^{-1}$. The second and third lines give the temperature and the visual extinction corresponding to the local emissivity maximum. Numbers in parentheses refer to power of ten.

We have derived the main properties of the atomic to molecular deuterium transition for different densities and enhancement factors of the ISRF. The sensitivity of the results to the cosmic ray ionization rate, the formation rate on dust and the elemental deuterium abundance are studied. We extend the previous analytical expressions of the steady state abundance of $\mathrm{HD}$ in the case where $\mathrm{H}_{2}$ is prevailing but when photoprocesses are still dominant for HD.

The present model is valuable to interpret the observations of $\mathrm{H}_{2}$ and $\mathrm{HD}$ in a variety of physical conditions. We have shown that $N(\mathrm{HD}) / 2 N\left(\mathrm{H}_{2}\right)$ is a lower limit to the elemental deuterium abundance in diffuse and translucent clouds whereas $N(\mathrm{D}) / N(\mathrm{H})$ leads to an upper limit. So, the recent detections of HD with FUSE should be analyzed with care. The intensities of the pure rotational transitions are predicted for a large range of density and enhancement factors of the ISRF. The values are compatible with the detection of the $112 \mu \mathrm{m}$ transition in the Orion Bar by Wright et al. (1999). It is also shown that the intensity of the $56 \mu \mathrm{m}$ transition is below that of the $112 \mu \mathrm{m}$ transition for density below $10^{5} \mathrm{~cm}^{-3}$ and $\chi \leq 10^{5}$.
Up to now, this transition has not been detected by ISO. The future HERSCHEL mission will allow to search for HD $112 \mu \mathrm{m}$ at a higher sensitivity. A natural extension of our model is to include the vibrational excitation of HD. Detection of the 1-0 R(5) transition of HD at $2.46 \mu \mathrm{m}$ by Ramsay Howat et al. (2002) is interpreted as a result of chemical excitation. This could be tested in such models. However the fast vibrational radiative decay of HD of several $10^{-5} \mathrm{~s}^{-1}$ implies critical densities at least of the order of $10^{10} \mathrm{~cm}^{-3}$ and the vibrational level populations will be sub-thermal in most cases. It is therefore not surprising that vibrationally excited HD has not been detected in the Hubble Space Telescope (HST) spectra towards HD39603, a PDR region of density of several $10^{4} \mathrm{~cm}^{-3}$ in the NGC2023 nebula whereas a rich spectrum of vibrationnally excited hydrogen has been obtained by Meyer et al. (2001).

Future efforts will also be put on MHD shock models following the treatments developed for $\mathrm{H}_{2}$ (Wilgenbus et al. 2000). The detection of the $0-0 \mathrm{R}(5)$ transition of $\mathrm{HD}$ at $19.43 \mu \mathrm{m}$ by Bertoldi et al (1999) in the OMC1 cloud is due to shock excitation.

Acknowledgements. We thanks Thomas Nodé Langlois who contributed at an early stage to the implementation of HD to the PDR model. We thanks also the referee for his thoughtful remarks which improved the manuscript considerably. Support from the Programme National du Milieu Interstellaire (PCMI) is gratefully acknowledged.

\section{References}

Abgrall, H., Roueff, E., \& Viala, Y. P. 1982, A\&AS, 50, 505

Abgrall, H., Le Bourlot, J., Pineau des Forêts, G., et al. 1992, A\&A, 253,525

Abgrall, H., Roueff, E., \& Drira, I. 2000, A\&AS, 50, 505

Abgrall, H., \& Roueff, E. 2002, in preparation

Allison, \& Dalgarno, A. 1970, Atom. Data, 1, 289

Bakes, E. L. O., \& Tielens, A. G. G. M. 1994, ApJ, 427, 822

Bertoldi, F., Timmermann, R., Rosenthal, D., Drapatz, S., \& Wright, C. 1999, A\&A, 346, 267

Black, J. H., \& Dalgarno, A. 1973, ApJ, 185, L101

Black, J. H., \& Dalgarno, A. 1976, ApJ, 203, 132

Bluhm, H., \& de Boer, K. S. 2002, A\&A, 379, 82

Bohlin, R. C., Savage, D., \& Drake, J. F. 1978, ApJ, 224, 132

Boissé, P., Le Petit, F., Pineau des Forêts, G., \& Roueff, E. 2002, A\&A, submitted

Draine, B. 1978, ApJS, 36, 595

Federman, S. R., Glassgold, A., \& Kwan 1979, ApJ, 227, 466

Federman, S. R., Weber, J., \& Lambert, D. L. 1996, ApJ, 463, 181

Ferlet, R., André, M., Hébrard, G., et al. 2000, ApJ, 538, L69

Fitzpartrick, E. L., \& Massa, D. 1990, ApJS, 72, 163

Flower, D. R., \& Roueff, E. 1999, MNRAS, 309, 833

Flower, D. R., Le Bourlot, J., Pineau des Forêts, G., \& Roueff, E. 2000, in $\mathrm{H}_{2}$ in space, ed. Combes and Pineau des Forêts (Cambridge University Press), 23

Galli, D., \& Palla, F. 1998, A\&A, 335, 403

Gerlich, D., Herbst, E., \& Roueff, E. 2002, submited to PSS.

Gry, C., Boulanger, F., Nehmé, C., et al. 2002, A\&A, in press

Hartquist, T. W., Black, J. H., \& Dalgarno, A. 1978, MNRAS, 185, 643

Hogerheijde, M. R., Jansen, D. J., \& van Dishoeck, E. F. 1995, A\&A, 294, 792

Hollenbach, D. J., \& Mac Kee, C. F. 1979, ApJ, 41, 555 
Hollenbach, D. J., \& Tielens, A. G. G. M. 1999, Rev. Mod. Phys., 71, 173

Jansen, D. J., Spaans, M., Hogerheijde, M. R, \& van Dishoeck, E. F. 1995, A\&A, 303, 541

Jura, M. 1975, ApJ, 197, 581

Lacour, S., Sonnentrucker, P., Ferlet, R., et al. 2001, AAS, 199.6510

Le Bourlot, J., Roueff, E., \& Viala, Y. 1987, A\&A, 188, 137

Le Bourlot, J., Pineau des Forêts, G., Roueff, E., \& Flower, D. 1993, A\&A, 267, 233

Le Bourlot, J., Pineau des Forêts, G., Roueff, E., Dalgarno, A., \& Gredel, R. 1995, ApJ, 449, 178

Le Bourlot, J. 2000, A\&A, 360, 656

Lee, H. H., Herbst, E., Pineau des Forets, G., Roueff, E., \& Le Bourlot, J. 1996, A\&A, 311, 390

Lemoine, M., et al. 1999, New Astron., 4, 231

Le Petit, F., \& Roueff, E. 2002, in The Dissociative Recombination of Molecules with Electrons, ed. S. Guberman (Kluwer Academic, Plenum Publisher), to be published

Linsky, J. L., Diplas, A., Wood, B. E., et al. 1995, ApJ, 541, 335

Lis, D., Roueff, E., Gerin, M., et al. 2002, ApJ, 571, L55

Loinard, L., Castets, A., Ceccarelli, C., et al., A\&A, 357, 1169

Mathis, J. S., Rumpl, W., \& Nordsieck, K. H. 1977, ApJ, 217, 425

Meyer, D. M., Cardelli, J. A., \& Sofia, U. J. 1997, ApJ, 490, L103

Meyer, D. M., Jura, M., \& Cardelli, J. A. 1998, ApJ, 493, 222

Meyer, D. M., Lauroesch, J. T., Sofia, U. J., \& Bertoldi, F. 2001, ApJ, 553, L59

Parmar, P. S., Lacy, J. H., \& Achtermann, J. M. 1991, ApJ, 372, L25

Petitjean, P., Srianand, R., \& Ledoux, C 2000, A\&A, 364, L26
Pineau des Forêts, G., Roueff, E., \& Flower, D. R. 1989, MNRAS, 240,167

Rachford, B., et al. 2001, ApJ, 555, 839

Ramsay Howat, S. K., Timmermann, R., Geballe, T. R., Bertoldi, F., \& Mountain, C. M. 2002, ApJ, 566, 905

Roberts, H., \& Millar, T. J. 2000, A\&A, 361, 388

Roberts, H., \& Millar, T. J. 2000, A\&A, 364, 780

Roueff, E., \& Zeippen, C. J. 1999, A\&A, 343, 1005

Roueff, E., \& Zeippen, C. J. 2000, A\&AS, 142, 475

Roueff, E., Tiné, S., Pineau des Forêts, G., Falgarone, E., \& Gerin, M. 2000, A\&A, 354, L63

Roueff, E. 2002, in proceedings of the XIII IAP Coll.

Stancil, P. C., Lepp, S., \& Dalgarno, A. 1998, ApJ, 509, 1

Savage, B. D., \& Sembach, K. R. 1996, Ann. Rev. Astro. Astrophys., 34, 279

van der Tak, F. F. S., Schilke, P., Müller, H. S. P. 2002, A\&A, 388, L53

van Dishoeck, E. F., \& Black, J. H. 1986, ApJS, 62, 109

van Dishoeck, E. F., \& Black, J. H. 1988, ApJ, 334, 771

Varshalovich, D. A., Ivanchik, A. V., Petitjean, P., Srianand, R., \& Ledoux, C. L. 2001, AL, 27, 683

Viala, Y., Roueff, E., \& Abgrall, H. 1988a, A\&A, 190, 215

Viala, Y., Letzelter, C., Eidelsberg, M., \& Rostas, F. 1988b, A\&A, 193, 263

Wilgenbus, D., Cabrit, S., Pineau des Forêts, G., \& Flower, D. 2000, A\&A, 356, 1010

Wright, C. M., van Dishoeck, E. F., Cox, P., \& Kessler, M. F. 1999, ApJ, 515, L29 